\title{
Universal low-temperature ac conductivity of macroscopically disordered nonmetals
}

\author{
Jeppe C. Dyre \\ Institute of Mathematics and Physics, Roskilde University, P.O. Box 260, DK-4000 Roskilde, Denmark
}

(Received 27 April 1993)

\begin{abstract}
This paper discusses a macroscopic model for ac conduction in electronically or ionically conducting disordered solids. The model considers ac conduction in an inhomogeneous solid that is characterized by a spatially randomly varying thermally activated (frequency-independent) conductivity. Discretizing Maxwell's equations leads to an equivalent electrical circuit that is a simple-cubic lattice where each pair of nodes are linked by a resistor and a capacitor in parallel. The values of the resistors are determined by the local resistivity while the capacitors are all equal, given by the infinite-frequency dielectric constant. It is shown that the capacitor currents are Maxwell's displacement currents. Assuming uncorrelated resistances, the model is solved analytically at low temperatures in the effective-medium approximation (EMA) and in a naive percolation-path approximation. Both approximations predict similar universal ac responses as $T \rightarrow 0$, where the macroscopic frequency-dependent conductivity becomes independent of the activation-energy probability distribution. The universality represents an unusual type of regularity appearing in the extreme disorder limit. The universality prediction is tested by computer simulations of $200 \times 200$ lattices in two dimensions and of $50 \times 50 \times 50$ lattices in three dimensions. The computer simulations show that the EMA works very well in two dimensions in the whole temperature range studied; in particular, the low-temperature universality prediction is confirmed. In three dimensions the universality prediction is confirmed as well.
\end{abstract}

\section{INTRODUCTION}

Alternating current conduction in disordered solids has been studied during the last 40 years. ${ }^{1-7}$ Numerous papers have appeared, especially after 1970, reporting the frequency and temperature dependence of the electrical conductivity in electronically or ionically conducting disordered solids like glasses or various forms of imperfect crystals. With modern frequency analyzers the measurements are fast and fairly straightforward. A considerable amount of work has gone into developing theories of ac conduction, with the main focus on hopping models. ${ }^{8-10}$ Despite this, it is still not clear what the correct model is for ac conduction (in particular, whether macroscopic or microscopic inhomogeneities are responsible for the frequency dispersion), and it is unknown when and if Coulomb interactions are important. ${ }^{11,12}$ Consequently, the interpretation of data is highly subjective and few examples exist of ac measurements yielding unambiguous information about charge carrier motion in a bulk disordered solid.

Besides the lack of understanding of ac phenomena, there is another problem with the application of impedance spectroscopy to disordered solids: These solids show remarkably similar behavior in regard to their frequency-dependent conductivity and its temperature dependence. ${ }^{1-3,13}$ Thus, all disordered solids have an ac conductivity which depends on frequency as an approximate power law where the exponent is less than but close to one and goes to one as the temperature goes to zero. Furthermore, one observes in all cases a much less pronounced temperature dependence of the ac conductivity than that of the dc conductivity.

The present paper discusses a macroscopic model for ac conduction. The model, which is conceptually simpler than the popular hopping models, investigates the ac consequences of a spatially varying electrical conductivity. The model is based on the well-known MaxwellWagner effect, i.e., the fact that inhomogeneities give rise to a frequency dependence of the conductivity because charge carriers accumulate at the boundaries to less conducting regions, thereby creating dipolar polarization. While a number of papers have discussed the dc conductivity of disordered solids with macroscopic inhomogeneities, little work has gone into studying the ac aspects. In this paper, that extends and details a recent paper, ${ }^{14}$ the model is derived from Maxwell's equations, assuming the local conductivity is thermally activated. It is shown how to discretize the model and two approximations are applied, focusing on the low-temperature limit of the model. Both approximations predict a universality of the ac response as $T$ goes to zero. This prediction is confirmed by computer simulations in two and three dimensions.

The paper is organized as follows. In Sec. II, a brief review is given of the experimental observations and of the models hitherto studied. In Sec. III, the macroscopic model is formulated and discretized, and in Sec. IV, two analytical approximations are applied to the model, the effective-medium approximation (EMA) as well as a naive percolation path analysis. In Sec. V, the results from extensive computer simulations are reported, and finally Sec. VI contains a discussion.

\section{THE PHYSICS OF ac CONDUCTION IN DISORDERED SOLIDS}

The first systematic works on ac conduction in disordered solids were the "dielectric" studies of ionic conduc- 
tive oxide glasses. ${ }^{15-17}$ Soon after, in 1961, Pollak and Geballe $^{18}$ reported ac measurements on $n$-type doped crystalline silicon at helium temperatures (where the disorder due to the random substitution of the dopants becomes important). Only much later was the similarity between the ac response of ionic glasses and of electronically conducting disordered solids noted. ${ }^{1-3,13}$ During the last 20 years a large number of publications have reported ac measurements on disordered solids like amorphous semiconductors, ${ }^{3,4,19}$ fast ionic conductors, ${ }^{5,7}$ nonstoichiometric or polycrystals, ${ }^{20,21}$ ionic or electronically conducting polymers, ${ }^{22,23}$ metal-cluster compounds, ${ }^{24}$ polaronically conducting transition-metal oxides, ${ }^{3,25}$ organic semiconductors, ${ }^{26}$ or high-temperature superconductors above $T_{c}{ }^{27}$

Experimental data are usually reported in terms of the real part $\sigma^{\prime}(\omega)$ of the frequency-dependent conductivity $\sigma(\omega)=\sigma^{\prime}(\omega)+i \sigma^{\prime \prime}(\omega)$. There are, however, alternatives to this means of representation. Early publications on ionic glasses presented data in terms of the negative imaginary part $\epsilon^{\prime \prime}(\omega)$ of the complex dielectric constant $\epsilon(\omega)=\epsilon^{\prime}(\omega)-i \epsilon^{\prime \prime}(\omega)$ defined by

$$
\epsilon(\omega)=\frac{\sigma(\omega)-\sigma(0)}{i \omega} .
$$

Presently, data for ionic systems are often given in terms of the electric modulus $M(\omega)$ defined $^{28}$ by $M(\omega)$ $=i \omega / \sigma(\omega)$ (though it has been argued that this is not a good means of presenting data). ${ }^{29,30}$ Finally, there is also the possibility of using the complex resistivity $\rho(\omega)=1 / \sigma(\omega)$. $^{31}$

As mentioned above, all disordered solids exhibit the same qualitative ac behavior: Around the dielectric loss peak frequency marking the maximum of $\epsilon^{\prime \prime}(\omega), \omega_{m}$, $\sigma^{\prime}(\omega)$ starts to increase, and for $\omega \gg \omega_{m}, \sigma^{\prime}(\omega)$ follows an approximate power law: $\sigma^{\prime}(\omega) \propto \omega^{n^{\prime}}$. This behavior continues right up to phonon frequencies where the conductivity around $\omega=10^{12} \mathrm{~Hz}$ is of order $1(\Omega \mathrm{cm})^{-1.32}$ The signature of a power law is a straight line in a log-log plot. There is some controversy as to whether the observed power laws are truly fundamental ${ }^{1,33-35}$ or just an approximate description. 8,30 In any case, the exponent $n^{\prime}$ is always between 0.7 and 1.0 (the only exception seems to be one-dimensional conductors), ${ }^{36,37}$ and one always finds that $n^{\prime}$ goes to one as the temperature goes to zero. The ac conductivity is always less temperature dependent than the dc conductivity (when viewed in the usual log$\log$ plot), and for $T \rightarrow 0$ the ac conductivity becomes almost temperature independent. The dc conductivity usually follows an Arrhenius law. An important universal observation is the Barton-Nakajima-Namikawa (BNN) relation, ${ }^{38-42}$

$$
\sigma(0)=p \Delta \epsilon \omega_{m},
$$

where $\Delta \epsilon$ is the dielectric loss strength, $\Delta \epsilon=\epsilon^{\prime}(0)$ $-\epsilon^{\prime}(\infty)$, and $p$ is a numerical constant of order 1 . Since $\Delta \epsilon$ depends only weakly on temperature, the BNN relation implies that the activation energy of $\sigma(0)$ is equal to that of $\omega_{m} \cdot{ }^{17}$ In the majority of disordered solids $\sigma^{\prime}(\omega)$ obeys the time-temperature superposition principle, i.e., the fact that at different temperatures one observes the same function $\sigma^{\prime}(\omega)$ just scaled (i.e., displaced in the log$\log$ plot). This, in conjunction with the $\mathrm{BNN}$ relation and the Debye law $\Delta \epsilon \propto T^{-1}$, shows that the dimensionless conductivity $\widetilde{\sigma}=\sigma(\omega) / \sigma(0)$ is a function of $\omega /[T \sigma(0)] .^{43-45}$ A convenient name for the fact that all disordered solids show the same qualitative ac behavior is to refer to it as "quasiuniversality.",44

The early experiments on ac properties of ionic glasses were interpreted in terms of a distribution of relaxation times for associated Debye processes, as is common for dielectric relaxation in liquids. ${ }^{46}$ Workers in semiconductor physics in the 1960s proposed the pair approximation as a model for the ac loss. ${ }^{47,48}$ This model assumes the loss is due to independent pairs of sites in the solid, where each pair provides two possible positions for a localized electron. Mathematically, this corresponds to the description in terms of Debye processes in parallel that was used in the early work on ionic glasses.

In the pair approximation there is no dc conduction. This has to be assumed to be derived from a completely different process, whereby the $\mathrm{BNN}$ relation becomes very hard to understand. Furthermore, the pair approximation cannot explain the fact that the exponent $n^{\prime}$ goes to one as $T \rightarrow 0$. A version of the pair approximation, the correlated barrier hopping model, has been proposed by Elliott. 6,49 This model explains the low-temperature behavior of $n^{\prime}$ as a consequence of Coulomb force controlled variable range hopping and the model has a nonzero dc conductivity because the pairs are not isolated from each other.

A number of authors have considered phenomenological and intuitive models based on networks composed of resistors and capacitors. ${ }^{11,21,28,34,50-55}$ In the present paper, following Springett, Webmann et al., Sinkkonen, and Fishchuk, ${ }^{56-59}$ a resistor-capacitor network is also arrived at, but here it is derived directly from Maxwell's equations.

In the last 15 years a number of models have appeared $^{1,33-35,60-62}$ emphasizing the power-law behavior of $\sigma^{\prime}(\omega)$ which is regarded as fundamental, much like the power laws for second-order phase transitions found close to $T_{c}$. Thereby, fractal aspects of the conduction process are emphasized. ${ }^{35,63}$ Power laws also result if it is assumed that the phase difference between field and current is frequency independent. ${ }^{64}$

The most thoroughly studied models for ac conduction in disordered solids are probably the so-called hopping models. ${ }^{8-10}$ A hopping model considers the random walk of (usually) independent charge carriers in a disordered structure. If the charge carrier sites are marked $\mathbf{s}$ and $\Gamma\left(\mathbf{s} \rightarrow \mathbf{s}^{\prime}\right)$ denotes the rate for jumps from site $\mathbf{s}$ to site $\mathbf{s}^{\prime}$, a hopping model is characterized by the following master equation for the probability of finding a charge carrier at site $\mathbf{s}, P(\mathbf{s}, t)$,

$$
\frac{\partial P(\mathbf{s}, t)}{\partial t}=-\sum_{\mathbf{s}^{\prime}} \Gamma\left(\mathbf{s} \rightarrow \mathbf{s}^{\prime}\right) P(\mathbf{s}, t)+\sum_{\mathbf{s}^{\prime}} \Gamma\left(\mathbf{s}^{\prime} \rightarrow \mathbf{s}\right) P\left(\mathbf{s}^{\prime}, t\right) .
$$

The jump rates that are commonly taken to be an ex- 
ponential function of an activation energy and/or a tunneling distance, are usually assumed to vary randomly and to be nonzero only for nearest-neighbor jumps. The frequency-dependent conductivity is calculated from the Kubo formula ${ }^{65,66}$ that in $D$ dimensions is

$$
\sigma(\omega)=\lim _{V \rightarrow \infty} \frac{1}{D k_{B} T V} \int_{0}^{\infty}\langle\mathbf{J}(0) \cdot \mathbf{J}(t)\rangle e^{-i \omega t} d t
$$

where $\mathbf{J}$ is the total current in the volume $V$.

Hopping models are complex and cannot be solved analytically. To evaluate $\sigma(\omega)$ either one has to computer simulate, or to use some analytical approximation. An early approximation was the continuous time random walk (CTRW) approximation of Scher and Lax. ${ }^{43}$ Today the CTRW is regarded as the simplest available approximation, a mean-field Hartree-type approximation ${ }^{67}$ (note that the original derivation that converted the disordered Markovian hopping model to a non-Markovian random walk in a homogeneous medium was inconsistent). ${ }^{30,68}$ The standard approximation for disordered systems, which is often also used for hopping models, is the effective-medium approximation (EMA). ${ }^{9,67,69-71}$ A related approach is the extended pair approximation (EPA) of Summerfield and Butcher. ${ }^{72}$

Hopping models usually assume noninteracting charge carriers. Thus, the self-exclusion effect (allowing at most one particle at each site $^{73}$ ) is ignored, as well as are Coulomb interactions between the charge carriers. Recent work includes these effects, ${ }^{12}$ but at the price that the model becomes very complex and can only be studied by means of computer simulations. The macroscopic model considered in the next section includes Coulomb interactions via Gauss' law, without becoming extremely complex.

\section{THE MACROSCOPIC MODEL AND ITS DISCRETIZATION}

This section deals with setting up the equations governing ac conduction in a solid with a spatially varying (frequency-independent) conductivity. ${ }^{14,56-59}$ It is assumed that the solid has free charge carriers characterized by a local conductivity denoted by $g(\mathbf{r})$, as well as bound charges described by the spatially constant dielectric constant $\epsilon_{\infty}$, equal to the $\omega \rightarrow \infty$ limit of $\epsilon(\omega)$ in Eq. (1). It is not entirely unproblematic to assume distinguishability between free and bound charge carriers in ac fields, ${ }^{74}$ but the assumption will be made here without further justification.

The quantity of interest is the macroscopic free charge carrier conductivity, defined as the ratio between the spatially averaged free charge current density and the spatially averaged electric field. If $\mathbf{D}$ denotes the displacement vector, $\mathbf{J}$ the free charge carrier current density, and $\phi$ the electrostatic potential, the basic constitutive equations are

$$
\begin{aligned}
& \mathbf{D}(\mathbf{r}, t)=-\epsilon_{\infty} \nabla \phi(\mathbf{r}, t) \\
& \mathbf{J}(\mathbf{r}, t)=-g(\mathbf{r}) \nabla \phi(\mathbf{r}, t) .
\end{aligned}
$$

These equations should be combined with Gauss' law

$$
\nabla \cdot \mathbf{D}(\mathbf{r}, t)=\rho(\mathbf{r}, t)
$$

(where $\rho$ is the free charge carrier density) and the continuity equation

$$
\dot{\rho}(\mathbf{r}, t)+\nabla \cdot \mathbf{J}(\mathbf{r}, t)=0 .
$$

In a periodically varying field all quantities are written as a factor $e^{i \omega t}$ times a function of space. Thus, the continuity equation becomes $i \omega \rho+\nabla \cdot J=0$. Substituting Eqs. (5) and (6) into this expression and introducing the "Laplace frequency"

$$
s=i \omega \epsilon_{\infty},
$$

one arrives at the following equation for the electrostatic potential:

$$
\boldsymbol{\nabla} \cdot\{[s+g(\mathbf{r})] \nabla \phi(\mathbf{r}, s)\}=0 .
$$

In terms of $\phi$ the average current density is given by

$$
\mathbf{J}(s)=\frac{1}{V} \int_{V} g(\mathbf{r})[-\nabla \phi(\mathbf{r}, s)] d \mathbf{r} .
$$

We now turn to the discretization of Eq. (9). ${ }^{14,59} \mathrm{~A}$ discretization is necessary for solving the equation numerically, but it is also useful for developing an intuition about the problem and arriving at approximate analytical solutions. The discretization will be performed in $D$ dimensions. It is assumed that the function $\phi(r, s)$ is known only at the points of a simple-cubic lattice with lattice constant $a$. If Eq. (9) is considered at the lattice point with coordinates $\left(n_{1} a, \ldots, n_{D} a\right)$, the first of the $D$ terms on the left-hand side becomes upon discretization (for simplicity only the first coordinate is written out explicitly, the remaining unchanged coordinates are $\left.n_{2} a, \ldots, n_{D} a\right)$ :

$$
\begin{aligned}
\frac{\partial}{\partial x_{1}}\left((s+g) \frac{\partial \phi}{\partial x_{1}}\right]\left(n_{1} a, s\right)=a^{-2} & \left\{s+g\left[\left(n_{1}+\frac{1}{2}\right) a\right]\right\}\left\{\phi\left[\left(n_{1}+1\right) a, s\right]-\phi\left(n_{1} a, s\right)\right\} \\
& \left.-\left\{s+g\left[\left(n_{1}-\frac{1}{2}\right) a\right]\right\}\left\{\phi\left(n_{1} a, s\right)-\phi\left[\left(n_{1}-1\right) a, s\right]\right\}\right) .
\end{aligned}
$$

There are $D-1$ other similar terms, and Eq. (9) becomes the condition that the sum of all $D$ terms is zero. Remembering the definition of $s$ [Eq. (8)], this zero sum requirement is recognized as the Kirchhoff current conservation law for a lattice where each link is a resistor and a capacitor in parallel (Fig. 1). Each link admittance $y$ is given by $y=K(s+g)$, where $K$ is a constant that is determined from requiring the correct continuum limit of the free charge current density $J$ : If the resistor current is $I_{R}, \mathrm{~J}$ is numerically given by $J=I_{R} / a^{D-1}$. On the 
other hand, if the potential drop across a link is denoted by $\Delta \phi$ one has $I_{R}=K g \Delta \phi$ and $J=g \Delta \phi / a$. Combining these equations we find $K=a^{D-2}$, so the link admittance is given by

$$
y=a^{D-2}(s+g) \text {. }
$$

The circuit of Fig. 1 is not a direct physical representation of the solid. This is because, while the resistor currents are indeed the true free charge currents, the capacitor currents are "ghost" currents that are not just the currents due to the actual displacement of the bound charge carriers. For instance, if $\epsilon_{\infty}=\epsilon_{0}$ (the vacuum permittivity), there are no bound charges but the capacitors are still important in the circuit. The correct interpretation of Fig. 1 is the following: In an external ac field the circuit determines the electrostatic potential. This potential in turn determines the free charge currents as those running through the resistors. Clearly, the capacitors give rise to a frequency dependence of the overall circuit admittance, but this is not the effect we are looking for. The frequency dependence of the free charge currents comes about only as an indirect effect of the capacitors because of their influence on the node potentials.

In the real solid the free charges accumulate at certain places. In Fig. 1, the role of the capacitors is to exactly

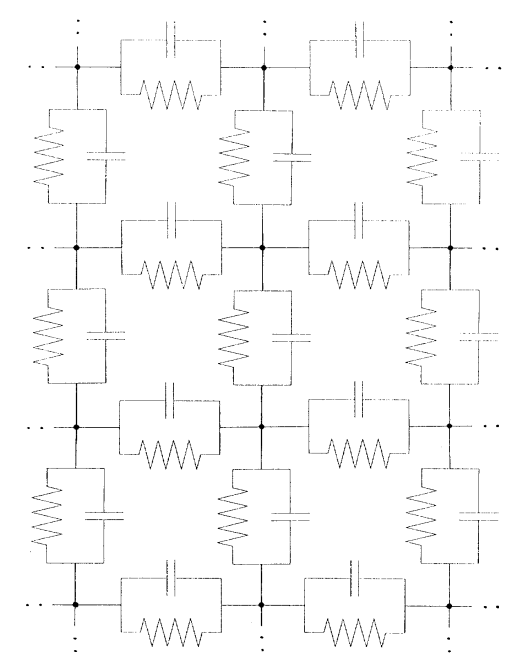

FIG. 1. Electrical equivalent circuit of Maxwell's equations discretized in 2D for an inhomogeneous conductor. Similar circuits exist in higher dimensions. All capacitors are equal while the resistors vary, reflecting the spatially varying conductivity of the solid. In the model studied here the resistors are assumed to be thermally activated and vary randomly and uncorrelated from link to link. In any external field the electrostatic potential is found from Kirchhoff's equations. The currents through the resistors are the free charge currents. The capacitor currents are Maxwell's displacement currents (parts of which are due to the bound charges and parts of which are "ghost" currents). The capacitor currents are nonzero in an ac field, thus allowing bound and free charge accumulation (the Maxwell-Wagner effect) without violating the Kirchhoff equation expressing the fact that there is no "total charge" (bound charge + free charge + "ghost" charge) accumulation at a node. compensate the free charge accumulation, so that there is no "total charge" accumulation at any node. It follows from Eq. (12) that the continuous analogue of the capacitor current is nothing but the well-known Maxwell displacement current $\mathbf{J}_{D}=\dot{\mathbf{D}}$. At first sight this may seem surprising since the displacement current is usually introduced in connection with completing Maxwell's equations to ensure that $\boldsymbol{\nabla} \cdot(\boldsymbol{\nabla} \times \mathbf{H})=0$. But this is done by adding to the free charge current $\mathbf{J}$ the term $\mathbf{J}_{D}=\dot{\mathbf{D}}$ so constructed that the divergence of $\mathbf{J}+\mathbf{J}_{D}$ is zero. The equation $\boldsymbol{\nabla} \cdot\left(\mathbf{J}+\mathbf{J}_{D}\right)=0$ follows from Eqs. (6) and (7); in an ac field this condition is nothing but Eq. (9).

The macroscopic frequency-dependent free charge conductivity may be calculated from the overall circuit admittance $Y(s)$. Here and henceforth the macroscopic free charge conductivity will be denoted by $\sigma(s)$, despite the risk of confusing it with the total conductivity appearing in Eq. (1). The latter quantity differs from the former by the factor $i \omega\left(\epsilon_{\infty}-\epsilon_{0}\right)$. In most experiments one looks for the real part of the conductivity only, and in any case it turns out that the $i \omega\left(\epsilon_{\infty}-\epsilon_{0}\right)$ term is insignificant in the present model at low temperatures and moderately low frequencies, which is the area of focus below.

Working in $D$ dimensions, the solid is discretized into $N^{D}$ points of a cubic lattice with sidelength $L=(N-1) a$. Two opposing faces of the cube are identified with the electrodes and short circuited. If the electrodes are subjected to a potential drop $\Delta \phi(s)$, the resulting current between the electrodes is given by $I(s)=Y(s) \Delta \phi(s)$. In order to calculate the macroscopic free charge conductivity from $Y(s)$ one has to subtract from $Y(s)$ the contribution due to the capacitor currents. Between the electrodes there are $N-1$ "layers" of parallel RC elements. The total current $I(s)$ is the same in each layer. Therefore, the sum of the resistor currents and the capacitor currents in the direction perpendicular to the electrodes is given by (with obvious notation)

$$
\sum I_{C}(s)+\sum I_{R}(s)=(N-1) I(s) .
$$

The sum of the capacitor currents is rewritten as a sum of $N^{D-1}$ terms where each term is the "one-dimensional" sum in the field direction (with obvious notation)

$$
\sum a^{D-2} s \Delta \phi(i \rightarrow i+1, s)=a^{D-2} s \Delta \phi(s) .
$$

Thus, Eq. (13) becomes

$$
\begin{aligned}
\sum I_{R}(s) & =(N-1) I(s)-N^{D-1} a^{D-2} s \Delta \phi(s) \\
& =\left[(N-1) Y(s)-N^{D-1} a^{D-2} s\right] \Delta \phi(s) .
\end{aligned}
$$

The macroscopic free charge conductivity is defined as the ratio between average free charge current density and average electric field. The former quantity is

$$
\sum I_{R}(s) /\left[a^{D-1} N^{D-1}(N-1)\right]
$$

and the latter is $\Delta \phi(s) /[(N-1) a]$. Using Eq. (14) we finally find

$$
\sigma(s)=\frac{N-1}{N^{D-1} a^{D-2}} Y(s)-s .
$$


For $N \rightarrow \infty$, Eq. (15) reduces to

$$
\sigma(s)=\frac{Y(s)}{L^{D-2}}-s
$$

For a given continuously varying local conductivity, $g(\mathbf{r})$, the discretization becomes exact for $a \rightarrow 0$. A few further assumptions are now made. First, it is assumed that the local conductivity is thermally activated and that the spatial variation in conductivity is due to the activation energy varying in space:

$$
g(\mathbf{r})=g_{0} e^{-\beta E(\mathbf{r})} .
$$

Here $\beta=1 /\left(k_{B} T\right)$. The activation energy is expected to vary because the local structure of the solid varies, leading to a varying mobility or perhaps to a varying internal electrostatic potential. ${ }^{59,75,76}$ In most cases one expects the activation energy to vary relatively little; however, our main focus here and below is the low-temperature limit where the local conductivity eventually varies several orders of magnitude.

It is realistic to assume a finite correlation length, $\xi$, for $E(\mathbf{r})$, where $\xi$ as usual is defined by

$$
\left\langle E(\mathbf{r}) E\left(\mathbf{r}^{\prime}\right)\right\rangle \simeq E_{0}^{2} e^{-\left|\mathbf{r}-\mathbf{r}^{\prime}\right| / \xi}, \quad\left|\mathbf{r}-\mathbf{r}^{\prime}\right| \rightarrow \infty .
$$

We now make an assumption which is very useful both from an analytical point of view (Sec. IV) and a numerical point of view (Sec. V): It is assumed that, by putting the lattice constant $a$ equal to $\xi$, correlations beyond $a$ may be ignored. ${ }^{57,77}$ The values of $g$ are thus assumed to be uncorrelated from link to link. In this approximation the problem is fully specified by the local activation energy probability distribution, $p(E)$, while details regarding how the activation energy varies in space are ignored.

Let us consider the low- and high-frequency limits of $\sigma(s)$ in the model. For $s \rightarrow 0$ the capacitors play no role and all circuit currents are free charge currents. Effectively, the circuit reduces to an ordinary resistor circuit. Such resistor circuits have been investigated extensively in the low-temperature limit. ${ }^{7-80}$ In this limit the current mainly follows the percolation paths giving the "easiest" ways between the electrodes. This picture is arrived at as follows. Imagine the resistors being removed from the lattice and then reintroduced in order of decreasing admittance. At a certain filling rate, the socalled link percolation threshold, infinitely large connect'ed clusters appear, creating a connection between the electrodes. (In two dimensions the link percolation threshold is given by $p_{c}=\frac{1}{2}$ exactly ${ }^{81}$ while simulations in three dimensions have shown that $p_{c}=0.2488 .{ }^{82}$ ) At low temperatures, adding further admittances beyond the percolation threshold does not change the overall circuit admittance significantly since the added admittances are much smaller than the admittances of the percolation cluster. Therefore, the total circuit admittance is dominated by the admittance of the percolation cluster that, in turn, is dominated by the smallest admittance on the cluster. This idea, which is now more than 20 years old, ${ }^{78-82}$ was later proved rigorously. ${ }^{83}$ At low temperatures one thus finds

$$
\sigma(0) \propto e^{-\beta E_{c}},
$$

where the percolation energy $E_{c}$ is defined by

$$
\int_{-\infty}^{E_{c}} p(E) d E=p_{c} .
$$

The high-frequency limit of the conductivity is straightforward to evaluate. For $s \rightarrow \infty$ the capacitors completely dominate the circuit. As a result the potential drop perpendicular to the electrodes is everywhere the same, corresponding to a uniform electric field, so the macroscopic conductivity is given by

$$
\sigma(\infty)=\langle g\rangle \text {. }
$$

In one dimension the circuit becomes particularly simple. Since the total circuit impedance is a sum of the impedances of RC elements, one finds if $g(E)=g_{0} e^{-\beta E}$

$$
\frac{1}{Y(s)}=(N-1) \int_{-\infty}^{\infty} \frac{p(E)}{a^{-1}[g(E)+s]} d E \text {. }
$$

Substituted into Eq. (15) this implies for $N \rightarrow \infty$ the following equation for $\sigma(s)$ :

$$
\frac{1}{\sigma(s)+s}=\int_{-\infty}^{\infty} \frac{p(E)}{g(E)+s} d E=\int_{0}^{\infty} \frac{p(g)}{g+s} d g \text {. }
$$

As a simple example consider the "box model," i.e., the case where $p(E)=1 / E_{0}\left(0<E<E_{0}\right)$. In that case the distribution of local admittances is (compare Appendix B )

$p(g)=p(E)\left|\frac{d E}{d g}\right|=\frac{1}{\beta E_{0}} \frac{1}{g} \quad\left(g_{0} e^{-\beta E_{0}}<g<g_{0}\right)$.

Equation (23) thus becomes

$$
\begin{aligned}
\frac{1}{\sigma(s)+s} & =\frac{1}{\beta E_{0}} \int_{g_{0} e^{-\beta E_{0}}}^{g_{0}} \frac{1}{g(g+s)} d g \\
& =\frac{1}{\beta E_{0}} \frac{1}{s} \ln \left[\frac{1+s /\left(g_{0} e^{-\beta E_{0}}\right)}{1+s / g_{0}}\right) .
\end{aligned}
$$

For large $\beta$ and low Laplace frequencies $\left(s \ll g_{0}\right)$ this reduces to

$$
\sigma(s)+s=\beta E_{0} \frac{s}{\ln \left[1+s /\left(g_{0} e^{-\beta E_{0}}\right)\right]} .
$$

Letting $s$ go to zero one finds that the de conductivity is given by

$$
\sigma(0)=\beta E_{0} g_{0} e^{-\beta E_{0}} .
$$

Thus, the dc conductivity activation energy is equal to the largest activation energy met on the one-dimensional (1D) path between the electrodes. This is the 1D analogue of Eq. (19).

According to Eq. (26) the conductivity becomes frequency dependent when $s$ is of order $s_{0}=g_{0} e^{-\beta E_{0}}$ $=\sigma(0) /\left(\beta E_{0}\right)$. Thus, at low temperatures the frequency dependence sets in already for $s<\sigma(0)$. At these low temperatures and moderate frequencies $s$ may be ignored in $\sigma(s)+s$, reflecting the fact that the capacitor currents are very small. Equation (26) may thus be written 


$$
\sigma(s)=\sigma(0) \frac{s / s_{0}}{\ln \left(1+s / s_{0}\right)} .
$$

In terms of the real frequency $\omega$ and the characteristic time $\tau=\epsilon_{\infty} / s_{0}$, Eq. (28) becomes

$$
\sigma(\omega)=\sigma(0) \frac{i \omega \tau}{\ln (1+i \omega \tau)}
$$

This equation was first derived for a hopping model. ${ }^{45}$ Note that, even though the capacitor currents for $\beta \rightarrow \infty$ are very small for a range of low frequencies, the capacitors may not be ignored from the circuit. If the capacitors are removed, there is no frequency dependence left. Thus, while the free charge currents run through the resistors and the capacitor currents are extremely small, the latter still have a very important effect on the magnitude of the average free charge currents, resulting in the dramatic frequency dependence of Eq. (29). In the numerical simulations reported below in two and three dimensions the same effect was found at low temperatures.

\section{TWO APPROXIMATE ANALYTICAL SOLUTIONS}

This section develops two analytical approximations for calculating $\sigma(s)$, focusing on the low-temperature region. In the $T \rightarrow 0$ limit a universality appears and (except for a scaling) $\sigma(s)$ becomes independent of the activation energy probability distribution $p(E)$.

The standard approximation for treating disordered systems analytically is the effective-medium approximation. ${ }^{77,84}$ In some contexts this approach is referred to as the coherent potential approximation (CPA). ${ }^{85,86}$ It has a number of desirable analyticity properties and seems to offer the best available compromise between being simple and being realistic. Here, the problem is to calculate the overall admittance of a large network whose admittances are independent random variables. The basic idea of the EMA is to focus on one particular admittance of the network, regarding it as placed in an "effective medium" with equal admittances $y_{m}$. The effective medium is constructed to best possibly mimic the average surroundings of the particular admittance. This is done by requiring that the electric field around the particular admittance on the average is equal to the distant homogeneous field of the surrounding effective medium, leading to the following equation for determining $y_{m}$ in $D$ dimensions ${ }^{77,87}$ (where sub $y$ implies an average over the admittance probability distribution)

$$
\left\langle\frac{y-y_{m}}{y+(D-1) y_{m}}\right\rangle_{y}=0 \text {. }
$$

The total network admittance $Y$ is found from $y_{m}$ (for $N \rightarrow \infty)$ via the obvious identity

$$
Y=N^{D-2} y_{m} \text {. }
$$

The EMA is exact in one dimension and it becomes exact for $D \rightarrow \infty .^{88}$ In the high-frequency limit the EMA is correct in all dimensions for the circuit of Fig. 1. In two dimensions the EMA is believed to be quite reliable; ${ }^{89}$ here it gives the correct percolation threshold $p_{c}=\frac{1}{2}$ and in a recent weak disorder perturbation calculation it was shown that the EMA is correct up to and including the fourth-order terms. ${ }^{90}$ These results in two dimensions are both consequences of the EMA satisfying the duality symmetry of the square lattice. ${ }^{91}$ In three dimensions the EMA is less reliable ${ }^{84,90}$ thus the EMA predicts $p_{c}=\frac{1}{3}$, whereas simulations yield $p_{c}=0.2488 .{ }^{82}$ Various improvements of the EMA exist ${ }^{92}$ but they are rather involved and will not be used here.

Combining Eqs. (12), (16), and (31) yields $y_{m}=a^{D-2}(\sigma+s)$. When this is substituted into Eq. (30) the EMA equation for the conductivity becomes ${ }^{59}$ [using Eq. (12)]

$$
\left\langle\frac{g-\sigma}{g+(D-1) \sigma+D s}\right\rangle_{g}=0 \text {. }
$$

For $s \rightarrow \infty$, Eq. (32) correctly gives $\sigma=\langle g\rangle$ [Eq. (21)] because the denominator becomes almost constant and may be ignored. Equation (32) may be solved numerically (Appendix A). In the next section the predictions of Eq. (32) at finite temperatures are compared to the results of simulations in 2D. Here we proceed to investigate the $T \rightarrow 0$ limit $^{14}$ where Eq. (32) implies a universal frequency dependence given as the solution of a simple transcendental equation.

Since $g-\sigma=g+(D-1) \sigma+D s-D(\sigma+s)$, Eq. may be rewritten as

$$
\frac{1}{D(\sigma+s)}=\left\langle\frac{1}{g(E)+(D-1) \sigma+D s}\right\rangle_{E},
$$

where the average is now over the activation energy distribution and $g(E)=g_{0} \exp (-\beta E)$. In the limit $\beta \rightarrow \infty$, $g(E)$ varies rapidly and for given $\sigma$ and $s$ there are essentially just two extreme possibilities, depending on $E$, either $g(E)<<(D-1) \sigma+D s$ or $g(E) \gg(D-1) \sigma+D s$. In the former case $g(E)$ may be ignored while in the latter case the denominator becomes very large and there is little contribution to the right-hand side. The energy separating the two cases, $E_{g}(s)$, is given by

$$
E_{g}(s)=-\frac{1}{\beta} \ln \left[\frac{(D-1) \sigma+D s}{g_{0}}\right]
$$

For large $\beta$, Eq. (33) thus becomes

$$
\frac{1}{D(\sigma+s)}=\frac{1}{(D-1) \sigma+D s} \int_{E_{g}(s)}^{\infty} p(E) d E
$$

or

$$
\frac{D-1}{D}+\frac{s}{D(\sigma+s)}=\int_{E_{g}(s)}^{\infty} p(E) d E .
$$

Subtracting from Eq. (36) the $s=0$ case of Eq. (36) itself leads to

$$
\frac{s}{D(\sigma+s)}=\int_{E_{g}(s)}^{E_{g}(0)} p(E) d E .
$$

For large $\beta, E_{g}(s)$ is close to $E_{g}(0)$ and the integral may be replaced by $p\left[E_{g}(0)\right]\left[E_{g}(0)-E_{g}(s)\right]$; thus, 


$$
\begin{aligned}
& \frac{s}{D(\sigma+s)}=-\frac{p\left[E_{g}(0)\right]}{\beta}\left[\ln \left[\frac{(D-1) \sigma(0)}{g_{0}}\right]\right. \\
&\left.-\ln \left[\frac{(D-1) \sigma+D s}{g_{0}}\right]\right] \\
&= \frac{p\left[E_{g}(0)\right]}{\beta} \ln \left[\frac{\sigma}{\sigma(0)}+\frac{D s}{(D-1) \sigma(0)}\right] .
\end{aligned}
$$

Introducing the dimensionless variables

$$
\widetilde{\sigma}=\frac{\sigma}{\sigma(0)}, \quad \widetilde{s}=\frac{\beta}{D p\left[E_{g}(0)\right] \sigma(0)} s,
$$

Eq. (38) for $\beta \rightarrow \infty$ reduces $^{14}$ to

$$
\widetilde{\sigma} \ln (\widetilde{\sigma})=\widetilde{s} .
$$

Equation (40) was previously derived for specific hopping models ${ }^{69,45}$ and, in the context of macroscopically inhomogeneous solids, it was derived by Fishchuk for the box distribution of activation energies. ${ }^{59}$ The importance of Eq. (40), however, as appears from the above derivation, lies in the fact that the equation is universal, completely independent of the activation energy probability distribution [an implicit assumption made above is that $p(E)$ is smooth around $\left.E_{c}\right]$. Note that Eq. (40) is only valid for $D>1$; for $D=1$ one has $E_{g}(0)=\infty$ and the step leading from Eq. (37) to Eq. (38) is invalid.

Figure 2(a) shows the dimensionless conductivity of Eq. (40) (solid curve) in a log-log plot for real dimensionless Laplace frequencies [the solution of Eq. (40) is discussed in Appendix A that also gives an analytical approximation to $\widetilde{\sigma}(\widetilde{s})]$. For large and real $\widetilde{s}$ the conductivity follows an approximate power law $\widetilde{\sigma} \propto \widetilde{s}^{u}$, where $u$ is about 0.9 in a large region. For large Laplace frequencies Eq. (40) roughly implies

$$
\widetilde{\sigma}_{\mathrm{EMA}}=\frac{\widetilde{s}}{\ln \left(\widetilde{\sigma}_{\mathrm{EMA}}\right)} \simeq \frac{\widetilde{s}}{\ln (\widetilde{s})},
$$

which, in turn, implies

$$
u=\frac{d \ln (\widetilde{\sigma})}{d \ln (\widetilde{s})} \simeq 1-\frac{1}{\ln (\widetilde{s})} \quad(\mathrm{EMA}, \widetilde{s} \gg>1)
$$

At real frequencies $\widetilde{s}$ is imaginary. Writing $\widetilde{s}=i \widetilde{\omega}$, Fig. 2(b) shows the real part $\widetilde{\sigma}_{\text {EMA }}^{\prime}(\widetilde{\omega})$ (solid curve) and the imaginary part $\widetilde{\sigma}_{\text {EMA }}^{\prime \prime}(\widetilde{\omega})$ (dashed curve) of the conductivity. At large frequencies these functions both follow approximate power laws. From the approximate expression

$$
\widetilde{\sigma}_{\mathrm{EMA}} \simeq \frac{i \widetilde{\omega}}{\ln (i \widetilde{\omega})}=\frac{i \widetilde{\omega}}{\ln (\widetilde{\omega})+i(\pi / 2)} \quad(\widetilde{\omega} \gg 1)
$$

one finds

$$
\widetilde{\sigma}_{\mathrm{EMA}}^{\prime} \simeq \frac{\pi}{2} \frac{\widetilde{\omega}}{\ln ^{2}(\widetilde{\omega})}, \quad \widetilde{\sigma}_{\mathrm{EMA}}^{\prime \prime} \simeq \frac{\widetilde{\omega}}{\ln (\widetilde{\omega})} \quad(\widetilde{\omega}>>1)
$$

This implies, for the approximate exponents defined by $\widetilde{\sigma} \sim \widetilde{\omega}^{n^{\prime}}$ and $\widetilde{\sigma}^{\prime \prime} \sim \widetilde{\omega}^{n^{\prime \prime}}$, $n^{\prime}=1-\frac{2}{\ln (\widetilde{\omega})}, \quad n^{\prime \prime}=1-\frac{1}{\ln (\widetilde{\omega})} \quad(\mathrm{EMA}, \widetilde{\omega}>1)$.

The conductivity at real Laplace frequencies, as well as both its real and imaginary parts taken at real frequencies, all become almost proportional to frequency as it goes to infinity. Note that this is not just a trivial effect reflecting conduction in the capacitors, since the capacitor currents do not contribute to the free charge conductivity. In fact, at any given temperature $\widetilde{\sigma}$ stabilizes and becomes frequency independent at sufficiently large frequencies [the frequency range where Eq. (40) is valid is
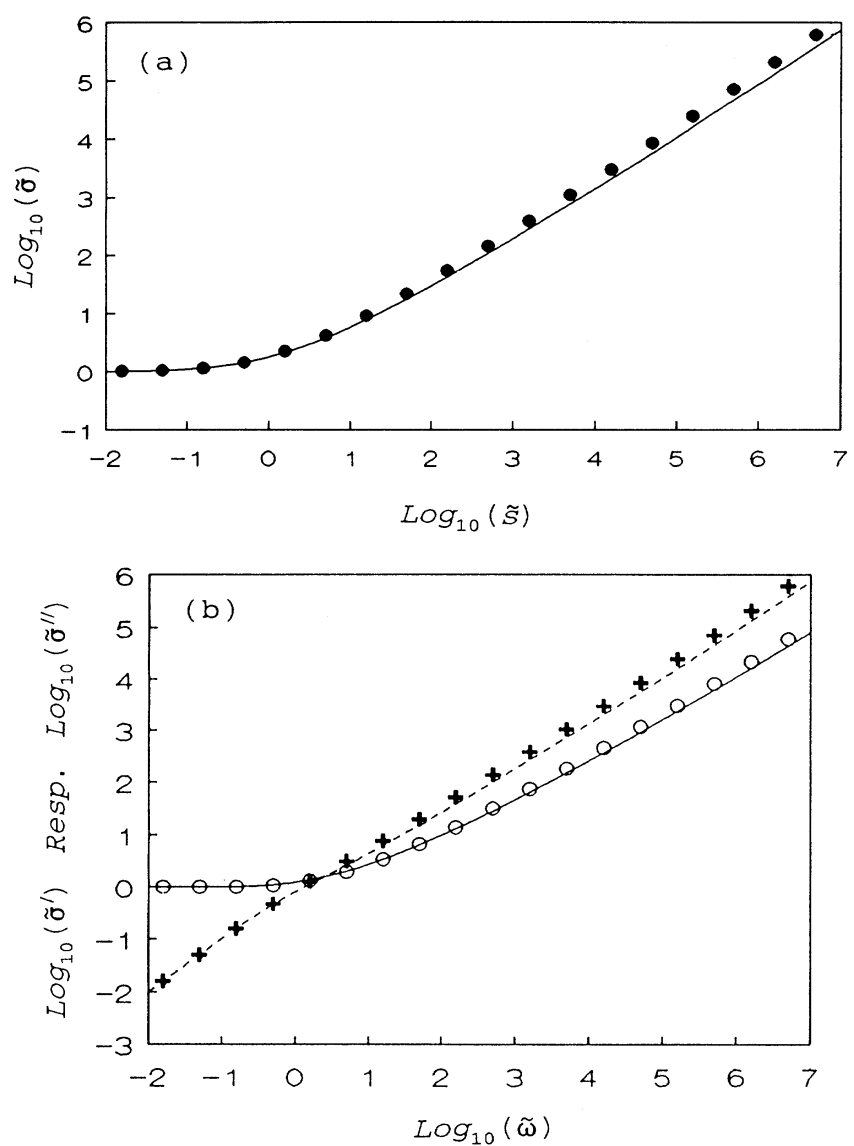

FIG. 2. Comparison of the predictions of the effectivemedium approximation (EMA) and the percolation-path approximation (PPA) (see the next page) for the low-temperature universal frequency-dependent conductivity which is independent of the activation energy probability distribution. (a) shows a log-log plot of the function $\widetilde{\sigma}_{\mathrm{EMA}}(\widetilde{S})$ at real dimensionless Laplace frequencies $\widetilde{S}[\mathrm{Eq} .(40)$, solid curve] and the function $\widetilde{\sigma}_{\mathrm{PPA}}\left(\widetilde{s}^{\prime}\right)$ [Eq. (47), dots], where $\widetilde{s}^{\prime}=2 \widetilde{s}$ scales the Laplace frequency so that the Taylor expansion of the two functions agree to first order at $\widetilde{s}=0$ (Ref. 45 ). (b) compares the real and imaginary parts of the two functions at real frequencies $\widetilde{\omega}=\widetilde{s} / i$, where $\widetilde{\sigma}_{\mathrm{EMA}}^{\prime}(\widetilde{\omega})$ is the solid curve, $\widetilde{\sigma}_{\mathrm{EMA}}^{\prime \prime}(\widetilde{\omega})$ is the dashed curve, $\widetilde{\sigma}_{\mathrm{PPA}}^{\prime}\left(\widetilde{\omega}^{\prime}\right)$ is given by the circles, and $\widetilde{\sigma}_{\mathrm{PPA}}\left(\widetilde{\omega}^{\prime}\right)$ is given by crosses $\left(\widetilde{\omega}^{\prime}=2 \widetilde{\omega}\right)$. The two approximations yield very similar predictions for the universal conductivity. In particular, one finds that their asymptotic behavior is identical as the frequency goes to infinity [Eqs. (42) vs (48) and (45) vs (50)]. 
only finite but becomes very large at low temperatures].

It is possible to throw light on the EMA solution by adopting a phenomenological point of view that makes sense in any dimension $D>1$ at sufficiently low temperatures. In this regime, the admittances of the network vary many orders of magnitude and the currents primarily follow the paths of least resistance, the "critical" or "percolation" paths. ${ }^{78,79}$ This is the idea leading to Eq. (19). We now propose an approximation referred to as the "percolation-path approximation" (PPA) that assumes that not only the dc currents but also the lowfrequency ac currents mainly follow the percolation paths. The solid is regarded as having several independently conducting "channels," each channel corresponding to a percolation path. This approximation ignores the complicated fractal nature of the percolation cluster. ${ }^{93}$ The problem of calculating the conductivity now becomes one-dimensional and one finds, as in Sec. III (where $K$ is an unknown numerical constant),

$$
\frac{1}{\sigma(s)+s}=K \int_{-\infty}^{E_{c}} \frac{p(E)}{g(E)+s} d E
$$

For a fixed range of frequencies around the transition frequency, the dominant contribution to Eq. (46) at low temperatures comes from energies close to $E_{c}$. Therefore, $p(E)$ may be replaced by $p\left(E_{c}\right)$ and the conductivity is the same as that of the one-dimensional box model already solved in Sec. III. Defining the dimensionless Laplace frequency $\widetilde{s}=s / s_{0}$, the PPA thus predicts [compare Eq. (28)]

$$
\widetilde{\sigma}_{\mathrm{PPA}}(\widetilde{s})=\frac{\widetilde{S}}{\ln (1+\widetilde{s})} .
$$

This function is plotted in Fig. 2(a) for real $\widetilde{s}$. The two solutions are very similar. As for the EMA solution one finds from Eq. (47) at real Laplace frequencies $\widetilde{\sigma} \sim \widetilde{s}^{u}$, where

$$
u=1-\frac{1}{\ln (\widetilde{s})} \quad(\text { PPA, } \widetilde{s}>>1) .
$$

At real frequencies $\widetilde{\omega}=\widetilde{s} / i$ one finds, since $\ln (1+i \widetilde{\omega})$ $=\ln \left(1+\widetilde{\omega}^{2}\right) / 2+i \arctan (\widetilde{\omega})$,

$$
\begin{aligned}
& \widetilde{\sigma}_{\mathrm{PPA}}^{\prime}(\widetilde{\omega})=\frac{\widetilde{\omega} \arctan (\widetilde{\omega})}{\ln ^{2}\left(1+\widetilde{\omega}^{2}\right) / 4+\arctan ^{2}(\widetilde{\omega})}, \\
& \widetilde{\sigma}_{\mathrm{PPA}}^{\prime \prime}(\widetilde{\omega})=\frac{\widetilde{\omega} \ln \left(1+\widetilde{\omega}^{2}\right)}{\ln ^{2}\left(1+\widetilde{\omega}^{2}\right) / 2+2 \arctan ^{2}(\widetilde{\omega})} .
\end{aligned}
$$

For $\widetilde{\omega} \rightarrow \infty$ one has $\widetilde{\sigma}_{\text {PPA }}^{\prime} \sim(\pi / 2) \widetilde{\omega} / \ln ^{2}(\widetilde{\omega})$ and $\widetilde{\sigma}_{\text {PPA }}^{\prime \prime} \sim \widetilde{\omega} / \ln (\widetilde{\omega})$ leading to the exponents

$n^{\prime}=1-\frac{2}{\ln (\widetilde{\omega})}, \quad n^{\prime \prime}=1-\frac{1}{\ln (\widetilde{\omega})} \quad($ PPA,$\widetilde{\omega} \gg 1)$.

These exponents are identical to those of the EMA [Eq. (45)].

Both the EMA and the PPA predict a universal frequency dependence of the low-temperature conductivity, independent of the activation energy probability distribution [assuming $p(E)$ is smooth around $E_{c}$ ]. Note that the two approximations yield similar predictions (Fig. 2), despite being derived from completely different points of view. The EMA, which is usually believed to be best for systems with weak disorder, ${ }^{88,90,94}$ has here been applied in the limit of extreme disorder. The PPA, on the other hand, only makes sense for the extreme disorder found at low temperatures. The similarity between the two approximations indicates that the EMA may be reliable even for systems of extreme disorder. On the other hand, the quantitative EMA prediction for the dc conductivity is known to be wrong in 3D because the percolation threshold is wrongly predicted, and thus one can, at most, expect the shape of the conductivity curve to be correct in the EMA. Only computer simulations can give reliable information as to whether universality really exists and, if it exists, whether it is well described by the two approximate theories.

\section{COMPUTER SIMULATIONS}

This section reports the results of computer simulations of the model in two and three dimensions. A lattice of admittances like in Fig. 1 is generated where each impedance is determined by an activation energy randomly chosen according to a probability distribution $p(E)$. Several different probability distributions were used; in Appendix B is explained how the activation energies were generated. At low temperatures large lattices are needed to obtain reasonable statistics. Even for relatively large lattices the system is not self-averaging at low temperatures and it is necessary to average over several lattices to obtain reproducible results. As a rule of thumb this procedure works well for $\beta<N$ whereas for larger $\beta$ the fluctuations become too large to be averaged out in a reasonable number of simulations. For simplicity all simulations are carried out at real Laplace frequencies only; by analytic continuation this is enough when one wants to compare the simulations to an analytical expression for the conductivity.

The calculation of the frequency-dependent conductivity may be performed by several methods. One possibility, the "brute force method," is to solve Kirchhoff's equations for the potential via some sparse matrix algorithm. Another possibility is to use Eq. (15) and calculate the overall circuit admittance between short-circuited electrodes by "elimination methods." These methods eliminate nodes of the lattice one by one by introducing new admittances without changing the overall circuit admittance, a process that is continued until one is left with only one admittance. The most general elimination method was introduced into the field by Fogelholm in $1980 ;^{95}$ it works as follows. Whenever a node is eliminated that has $n$ neighbors and the admittances $Y_{1}, \ldots, Y_{n}$ to its neighbors, all possible connections between the neighbors are introduced such that the $i$ th and the $j$ th neighbors are given the [additional] admittance $Y_{i} Y_{j} /\left(Y_{1}+\cdots+Y_{n}\right)$. For a full lattice the algorithm becomes very inefficient, but it works very well for calculations close to the percolation threshold where many admittances are zero. ${ }^{82}$ In two dimensions Frank and Lobb have developed a useful algorithm that eliminates nodes 
by working each of them towards the lower right via consecutive star-triangle transformations. ${ }^{96}$ By means of this algorithm it is possible to calculate the admittance of a $200 \times 200$ lattice in a few minutes on a modern PC.

Unfortunately no similarly efficient algorithm is available in 3D. Here it was found most efficient to use brute force methods. It is a rather complex numerical problem to solve large sparse systems of linear equations when the coefficients vary several decades. The standard GaussSeidel, as well as overrelaxation methods, ${ }^{97}$ converge too slowly. Fortunately, an algorithm has been developed, the algebraic multigrid (AMG) ${ }^{98,99}$ that was made precisely for problems of this type. The algorithm is an algebraic generalization of the standard geometric multigrid method used for solving elliptic differential equations. An excellent introduction to the multigrid idea, in general, has been given by Goodman and Sokal. ${ }^{100}$ The AMG solves a problem in a time only proportional to the number of equations. For an $N \times N$ lattice in 2D the computing time thus varies as $N^{2}$, for an $N \times N \times N$ lat- tice in $3 \mathrm{D}$ the time varies as $N^{3}$. The Frank-Lobb algorithm in $2 \mathrm{D}$ has a computing time varying as $N^{3}$; however, in practical applications it is still superior to the AMG because it avoids overflow problems and because the prefactors are clearly in its favor. It should be mentioned that other methods are also available. The transfer matrix method ${ }^{101}$ is an elimination method that works in all dimensions, and the Fourier acceleration brute force method ${ }^{102}$ is an alternative to the AMG for speeding up the Gauss-Seidel relaxation scheme.

Results of simulations in 2D of $100 \times 100$ lattices have been reported elsewhere ${ }^{14}$ for five activation energy probability distributions at the dimensionless inverse temperatures $\beta=4,16,64$. In Fig. 3 , these results are supplemented by simulations of $200 \times 200$ lattices at $\beta=5,10,20,40,80,160$ for the following activation energy distributions (see Appendix B): (a) asymmetric Gaussian, (b) symmetric exponential, (c) power law with exponent -4 , (d) triangle. For each temperature and activation energy distribution the figure shows the average of 10
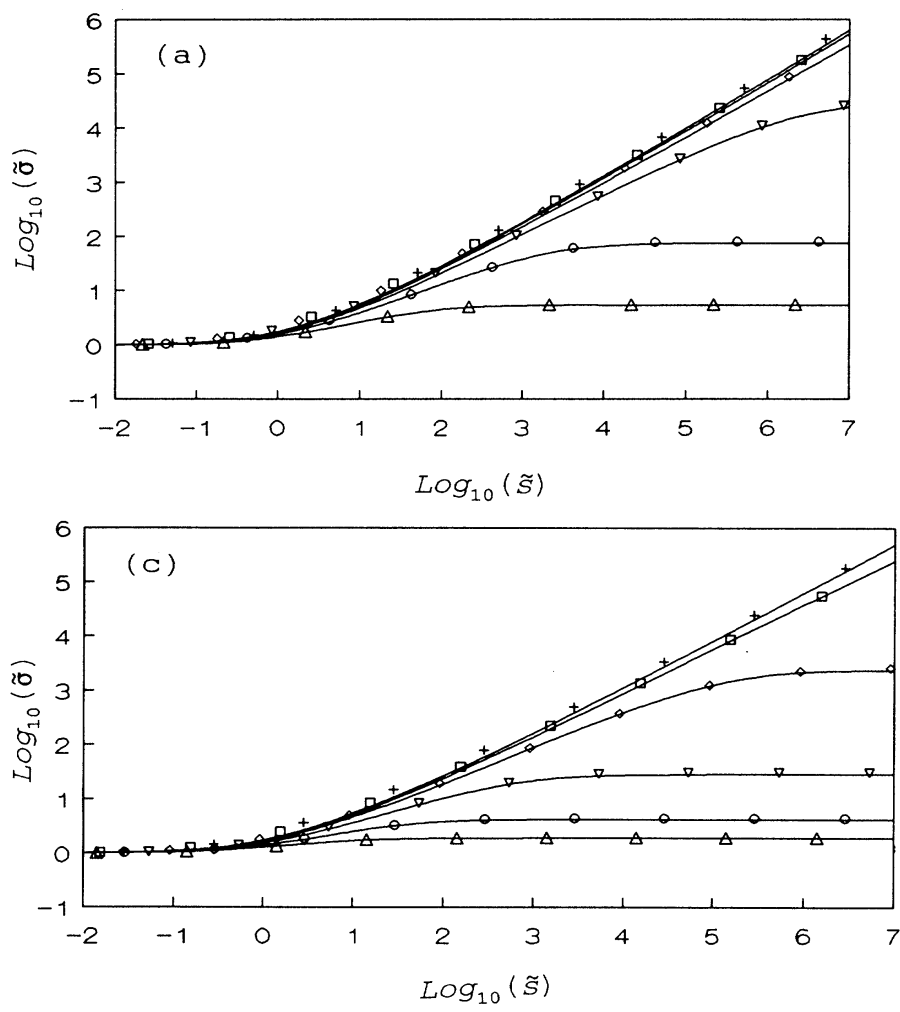
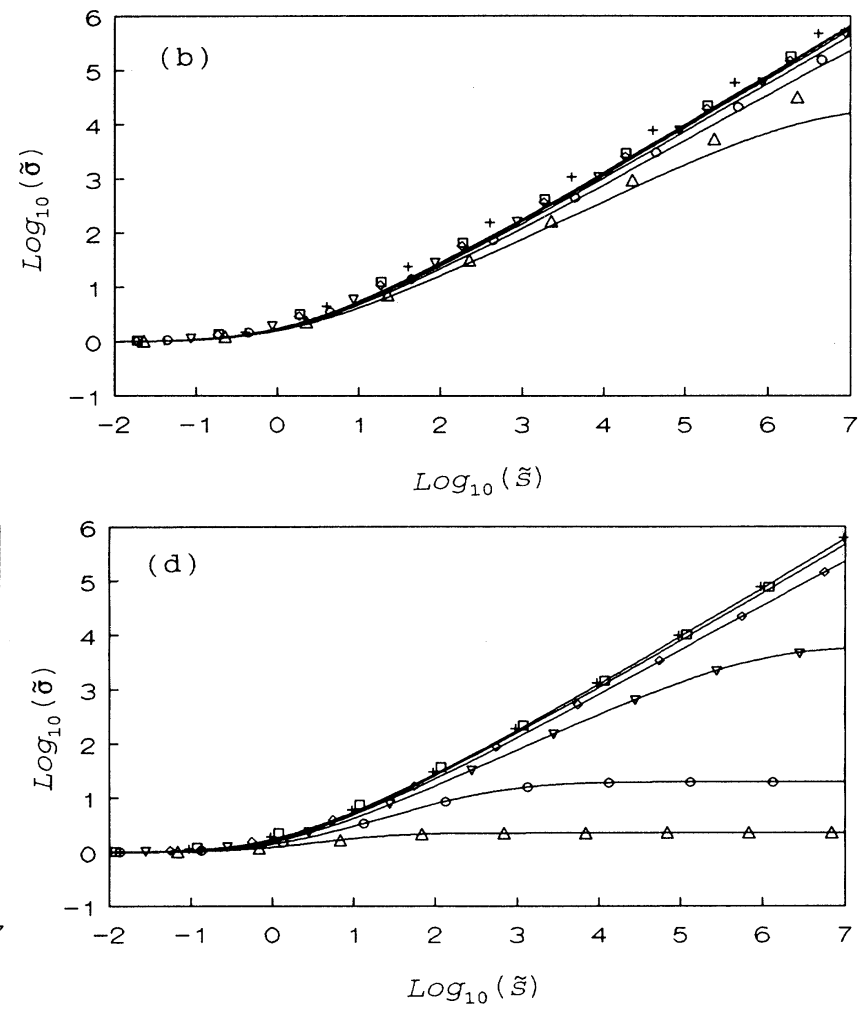

FIG. 3. Log-log plots of results of computer simulations in 2D for the dimensionless conductivity $\widetilde{\sigma}$ (points) compared to the EMA predictions (solid curves) at real dimensionless Laplace frequencies $\widetilde{s}$ [ $\widetilde{s}$ and $\widetilde{\sigma}$ are defined in Eqs. (8) and (39)]. Each figure shows the results of averaging 10 simulations of a $200 \times 200$ lattice solved by the Frank-Lobb algorithm (Ref. 96) and Eq. (15). Results for the following dimensionless inverse temperatures are shown: $\beta=5(\triangle), \beta=10(\bigcirc), \beta=20(\nabla), \beta=40(\diamond), \beta=80(\square), \beta=160(+)$, for the following activation energy probability distributions (Appendix B): (a) Asymmetric Gaussian, (b) symmetric exponential, (c) power law with exponent -4 , (d) triangle. In each case the distribution should be thought of as centered around an energy $\bar{E}$; this gives an extra factor $e^{-\beta \bar{E}}$ to the conductivity and scales the frequencies by the same amount, leaving both $\widetilde{\sigma}$ and $\widetilde{s}$ unchanged. The EMA predictions were found by solving Eq. (32) numerically (Appendix A). Given the fact that the EMA has no fitting parameters and that $\widetilde{s}$ is defined by scaling $s$ by a factor which is in some cases larger than $10^{50}$, the EMA provides a very good fit to the simulations. In particular, at low temperatures the frequency-dependent conductivity becomes universal both in the EMA and in the simulations. 
simulations. The solid curves are the EMA predictions found by solving Eq. (32) numerically (Appendix A). The EMA works well in $2 \mathrm{D}$ at all temperatures. In particular, one finds that universality is approached as $T \rightarrow 0$. Some of the low-temperature results deviate from the EMA prediction. However, given the fact that the frequency in some cases (at low temperatures) has been shifted more than 50 decades according in Eq. (39), the fit must be said to be satisfactory. The shape of the simulated frequency dependent conductivity at low temperatures is studied in Fig. 4 where the $\beta=80,160$ results from Fig. 3 have been replotted and supplemented by simulations of the Cauchy and the Box distribution. In Fig. 4, an empirical scaling of the Laplace frequency was allowed to fit the EMA universality prediction [Eq. (40)] in the best possible way.

In three dimensions results for averages of five $50 \times 50 \times 50$ lattices are shown in Fig. 5 for the values $\beta=10,30,60$ for the Cauchy and box activation energy
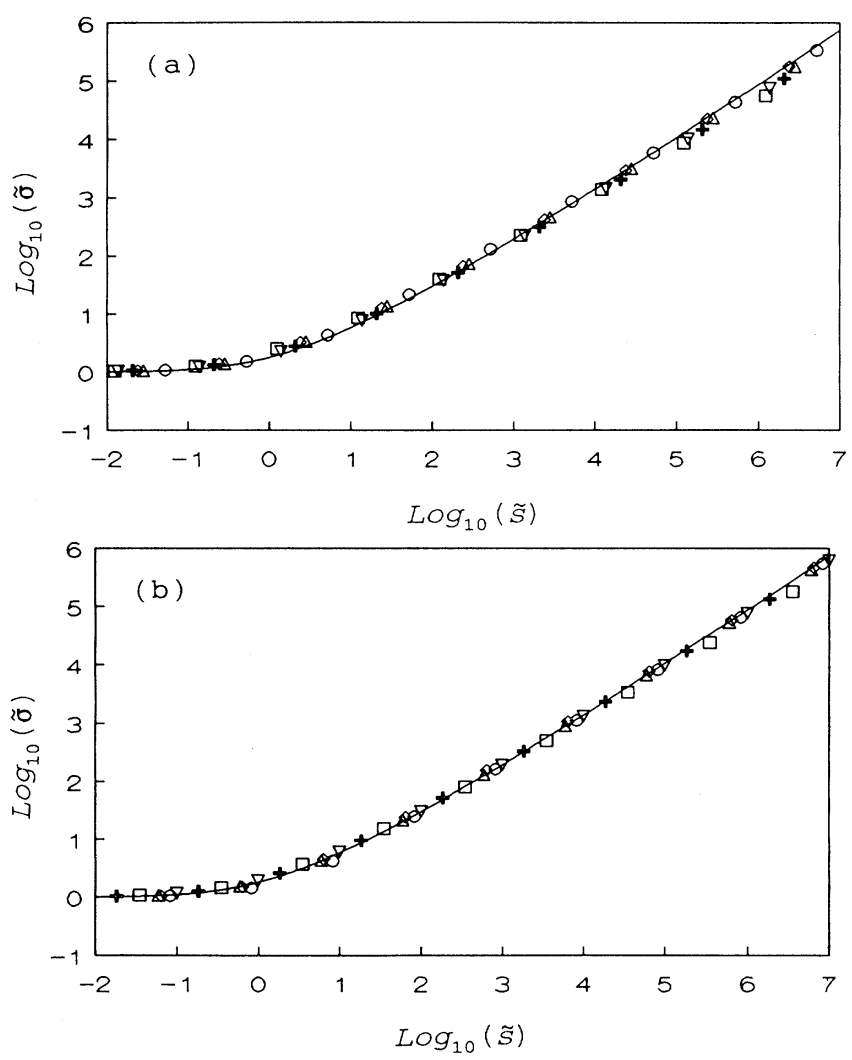

FIG. 4. Test of the EMA prediction at real dimensionless Laplace frequencies $\widetilde{s}$ for the low-temperature universal conductivity [solid curve, Eq. (40)] in log-log plots. An empirical rescaling of $\widetilde{s}$ has been allowed here to facilitate a comparison to the EMA prediction focusing only on the shape of $\widetilde{\sigma}(\widetilde{s})$. The figure shows results for $\beta=80$ (a) and $\beta=160$ (b) for asymmetric Gaussian $(\triangle)$, Cauchy $(O)$, symmetric exponential $(\diamond)$, power law with exponent $-4(\square)$, box $(+)$, triangle $(\nabla)$. As in Fig. 3 the results were obtained by averaging over 10 simulations of a $200 \times 200$ lattice in 2 D solved by means of the Frank-Lobb algorithm (Ref. 96) and Eq. (15). distributions. The conductivity was found by solving Kirchhoff's equations to determine the potential at each node and subsequently averaging all resistor currents. A potential $\phi=0$ was imposed at one electrode and $\phi=1$ at the other electrode; the remaining four faces of the cube were joined by imposing periodic boundary conditions. The 120000 equations for the node potentials were solved by means of the AMG1R5 FORTRAN algebraic multigrid subroutine available from the Yale multigrid library. ${ }^{103}$ This subroutine was found to be efficient, well documented, and providing useful error statements and warnings. The subroutine was previously successfully used for large
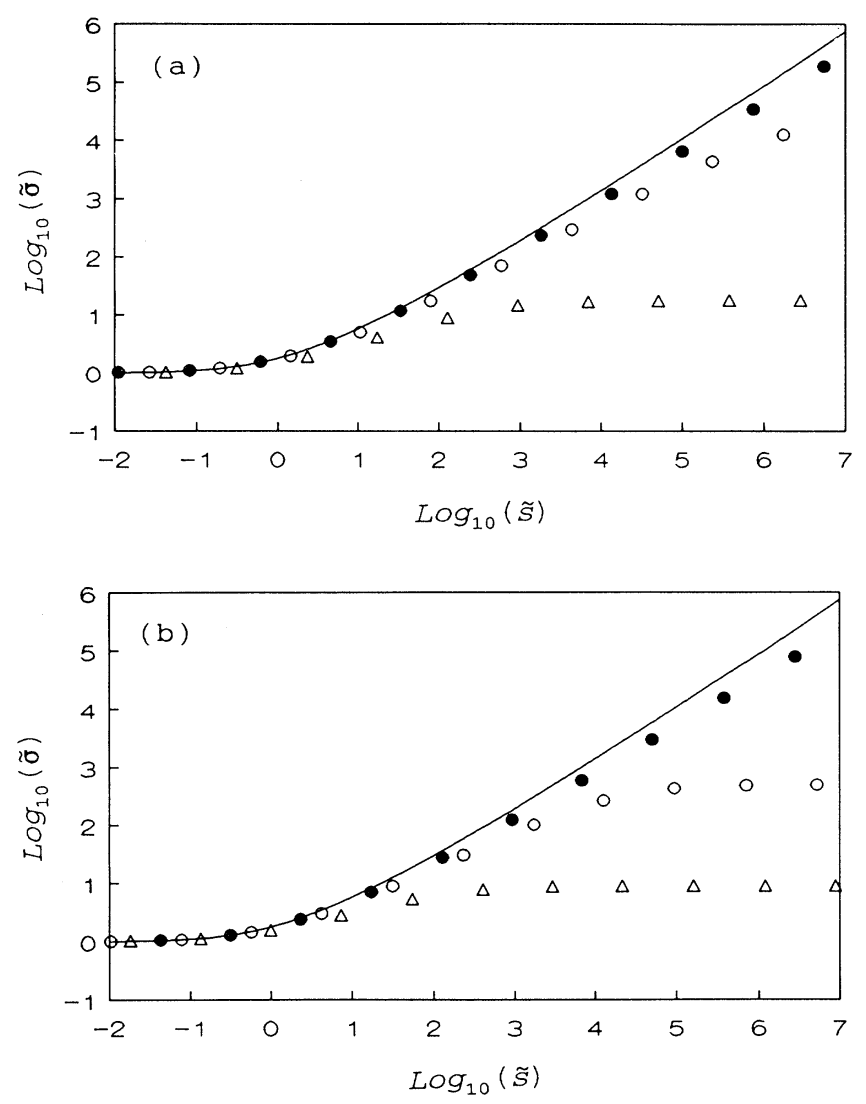

FIG. 5. Approach towards universality at low temperatures in three dimensions plotted in log-log plots. The figures show results for $\widetilde{\sigma}$ at real dimensionless Laplace frequencies $\widetilde{s}$ [both quantities are defined in Eqs. (8) and (39)] at $\beta=10(\triangle), \beta=30$ $(0)$, and $\beta=60(\bigcirc)$, for (a) the Cauchy and (b) the box distributions. Similar results were found for other distributions. Each point represents the average of five simulations of a $50 \times 50 \times 50$ lattice where Kirchhoff's equations were solved by the algebraic multigrid algorithm using the AMG1R5 Fortran subroutine (Ref. 103). The solid curve is the EMA prediction for the lowtemperature universal conductivity [Eq. (40)]. As in Fig. 4, an empirical rescaling of $\widetilde{s}$ was allowed, to focus on the shape of the conductivity curve only. Universality is approached at low temperatures, but unfortunately it was not possible to go to low enough temperatures to allow a detailed study of the exact shape of the universal conductivity in 3D. 
resistor network calculations by Edwards, Goodman, and Sokal. ${ }^{104}$ Still, the present problem is very complex because at low temperatures the coefficients of the linear equations vary several orders of magnitude. To avoid overflow and "division by zero" problems, the equations must be "massaged" somewhat. This was done by increasing the lowest $g$ 's to a standard low value, a procedure that is easily justified since these weak links carry little current anyway. The highest $g$ 's were also changed to a standard value by lowering them; this is allowed because they more or less short circuit their nodes anyway: they are not "bottlenecks" for the conduction process. In both cases, it was carefully checked that the calculated conductivity is independent of the cutoff's introduced, proving that the procedure it permissible. The calculations are rather demanding; the present work was carried out on a modern workstation with $128 \mathrm{MB}$ of memory (the AMG1R5 is quite memory consuming). The results are shown in Fig. 5 where they are compared to the EMA prediction (solid curve) as regards the predicted shape of the conductivity curve allowing an empirical frequency rescaling as in Fig. 4. Again one finds at low temperatures that the universal conductivity curve is approached. Similar results were found for four other activation energy probability distributions. ${ }^{4}$ Unfortunately, it was not possible to go to lower temperatures without serious overflow problems. At present it is therefore not possible to determine whether the universal conductivity curve in $3 \mathrm{D}$ is slightly less steep than in $2 \mathrm{D}$.

Finally, it should be mentioned that in both the 2D and the $3 \mathrm{D}$ simulations it was found that the capacitor currents at low temperatures are very small compared to the resistor currents in a wide range of frequencies around the transition frequency, thus confirming the analytical result for the $1 \mathrm{D}$ box model reported at the end of Sec. III.

\section{DISCUSSION}

This paper has investigated the ac consequences of having a spatially varying electrical conductivity, focusing on the low-temperature limit in the case when the local conductivity is thermally activated. The fact that spatial inhomogeneities give rise to polarization phenomena and therefore to a frequency dependence of the macroscopic conductivity has been known for many years. Despite this, little work has gone into studying ac aspects of the "macroscopic" model involving a range of local conductivities. For instance, no papers were given dealing with ac properties in this model of inhomogeneous media at the two "Conferences on Electrical Transport and Optical Properties of Inhomogeneous Media" held in 1978 and $1988 .^{105}$ Alternating current properties are instead usually modeled by means of hopping models where the disorder is assumed to be on the atomic scale. ${ }^{8-10,35,43-45}$ Hopping models usually do not include Coulomb interactions, a problem of recent concern, ${ }^{12}$ whereas the macroscopic model does include the effect of Coulomb interactions via Gauss' law.

The idea of a spatially randomly varying activation energy for the conductivity has been discussed for some time in connection with particular systems like heavily doped or compensated semiconductors ${ }^{59,75}$ or granular materials. ${ }^{76}$ For any disordered system one expects the various forms of inhomogeneities to influence the exact value of the activation energy of the local conductivity. The question is not whether or not this effect is present, but whether it is so pronounced that the concept of a local conductivity stops making sense, in which case the relevant model to use is a hopping model. At present it is not clear which disordered solids are best described by the macroscopic model and for which hopping models are best.

For a solid with a spatially varying conductivity, the relevant Maxwell's equations in a periodic external field boils down to the single equation (9) for the electrostatic potential. This equation is discretized by putting it on a cubic lattice. If the lattice constant $a$ is taken to zero, the discretization becomes exact. In Sec. III, however, the model was simplified further by choosing $a$ equal to the activation energy correlation length and by ignoring correlations beyond $a$. Thereby one ignores details of how the conductivity varies in space and the model becomes uniquely defined by the local activation energy probability distribution.

The discretization of Eq. (9) corresponds to the electric network of Fig. 1. Similar networks have been used many times before as models for the ac properties of disordered solids ${ }^{11,21,28,34,50-55}$ and the one-dimensional version of the network lies behind the electric modulus formalism. ${ }^{28}$ However, the network is traditionally used just as a suggestive picture of the solid and not justified from basic principles. When this is done (Sec. III) it turns out that the interpretation of the circuit is rather subtle. The network is not to be thought of as a straightforward representation of the solid. This is because the capacitor currents are Maxwell's displacement currents, parts of which do not involve real charge transport. In the model the free charge contribution to the conductivity depends on frequency only as an indirect effect of the capacitors: They influence the electrostatic potential that determines the resistor currents.

A simple model which may be solved exactly is the one-dimensional box model. In this model, as $\beta \rightarrow \infty$, there is a strongly frequency-dependent conductivity. It follows from Eq. (29) [compare Eq. (44)] that well above the transition frequency, the phase difference between the average field and the average resistor current is close to $\pi / 2$ [for any nonzero temperature Eq. (29) is only valid in a finite range of frequencies, but this range becomes very large at low temperatures]. This happens while at the same time the capacitor currents are very small compared to the resistor currents. The same peculiar effect of a strong frequency dependence of the average resistor currents simultaneously with very small capacitor currents was also observed in the computer simulations in $2 \mathrm{D}$ and $3 \mathrm{D}$.

The model was studied by computer simulations in $2 \mathrm{D}$ and $3 \mathrm{D}$. In $2 \mathrm{D}$, the results were fitted to the EMA prediction of Eq. (32). In $3 \mathrm{D}$ it is necessary to regard the scaling involved in the definition of the dimensionless Laplace frequency as a fitting parameter to obtain a reasonable fit. This is because the EMA has the percolation 
threshold wrong in 3D.

There are two conclusions to be drawn from the simulations. First, the simulations show that the frequency dependence of the conductivity indeed is universal at low temperatures. As far as is known to the author, the present work is the first time universality has been derived analytically and the first time universality has been seen in computer simulations of any model for ac conduction. The universality represents a new type of regularity valid for systems with extreme disorder. It should be noted, however, that, by letting the temperature go to zero, one actually moves into an unphysical domain since at temperatures of the order $10-30 \mathrm{~K}$ other processes such as tunneling would usually be expected to dominate the conduction. This has been ignored here.

In 1985 , Summerfield ${ }^{44}$ reported that several hopping models involving tunneling electrons solved in the extended pair approximation yield almost identical predictions for the frequency dependence of the conductivity. This phenomenon was referred to as "quasiuniversality." Since no simulations were reported it was not clear whether the effect is real or due to the approximation used, and there was no study of the temperature dependence of the conductivity for activated jump rates indicating an approach to true universality as $T \rightarrow 0$. Still, Ref. 44 gives the first hint to the existence of universality.

The second conclusion to be drawn from the simulations is that the EMA works very well in 2D at all temperatures. In $3 \mathrm{D}$ as $T \rightarrow 0$ the EMA also works well if a phenomenological rescaling is allowed to compensate for the fact that the EMA has the percolation threshold wrong. These results are highly nontrivial since doubt has often been expressed as to how reliable the EMA is for systems with a broad distribution of admittances. ${ }^{88,90,94}$ In one dimension the EMA universality prediction is not valid. But otherwise, since the EMA becomes exact for $D \rightarrow \infty,{ }^{88}$ there is reason to believe in Eq. (40) for all $D>1$ as a good approximation to the universal conductivity.

The percolation-path approximation is based on the fact that the percolation paths are preferred at low temperatures. These paths contain activation energies ranging up to a sharp cutoff at $E_{c}$ given by Eq. (20). Furthermore, in a fixed frequency range around the transition frequency it is clear that, at sufficiently low temperatures, only a narrow interval of activation energies are important (namely, those around the percolation energy determining the smallest admittances on the percolation paths). Therefore, the circuit admittance is expected to correspond to that of a one-dimensional solid with a sharp activation energy cutoff; this is why the PPA predicts the same frequency response as the one-dimensional box model. The fact that the PPA solution is very similar to the EMA solution shows that at sufficiently low temperatures a disordered solid, as a consequence of the underlying percolation, looks like a one-dimensional solid with a sharp cutoff in the activation energy distribution.

The PPA is not quite as straightforward as it seems at first sight. For very small $s$ the currents do follow the percolation paths, and as long as $s \ll g_{c}$, where $g_{c}$ is the smallest admittance on a percolation path, the conduc- tivity is clearly frequency independent. The conductivity starts to increase only for $s=s_{0}$, where $s_{0} \sim g_{c}$. But this is exactly the regime where the percolation picture starts to break down because all surrounding admittances also become of order $g_{c}$. In this range of frequencies the currents still mainly follow the percolation paths; however, when the current meets one of the poorest admittances on a percolation path, it may as well pass through the surrounding admittances. But since this happens seldomly, one expects the percolation-path calculation to be roughly valid, as confirmed by the simulations.

Since the dc conductivity is determined by the poorest admittances on a percolation path (of order $g_{c}$ ), the Laplace frequency for onset of ac conduction, $s_{0}$, is of order $\sigma(0)$. In particular, one expects $\sigma(0)$ and $s_{0}$ to have the same temperature dependence. This is consistent with the BNN relation [Eq. (2)]. The EMA and the PPA both predict that $s_{0}$ scales with $T \sigma(0)$ rather than with just $\sigma(0)$. This is sometimes referred to as "Summerfield scaling" 44 though it was first discussed by Scher and Lax in their papers on the continuous time random walk approximation. ${ }^{43}$ It is interesting to note that the extra factor $T$ in the Summerfield scaling of hopping models ${ }^{43-45}$ is derived from the $1 /\left(k_{B} T\right)$ factor of the fluctuationdissipation theorem Eq. (4) that is not applied in the macroscopic model.

What are the consequences of the presently reported results for the interpretation of experiments? First, it is to be noted that the EMA (or the PPA) $T \rightarrow 0$ universality prediction gives a rather good fit to many experiments. This has been shown in detail elsewhere ${ }^{45}$ where a boxtype hopping model yielding the same frequencydependent conductivity [Eqs. (40) and (47)] was discussed in detail. Thus, as in experiments ${ }^{45}$ Eq. (40) or (47) imply, (1) $\sigma^{\prime}(\omega)$ follows an approximate power law with an exponent $n^{\prime}$ less that but close to one, where $n^{\prime}(\omega)$ is weakly increasing and $n^{\prime} \rightarrow 1$ as $T \rightarrow 0$ (the latter is because, in a fixed frequency range, as the temperature goes to zero, one in effect measures further and further out on the universal conductivity curve); (2) when there is no detectable dc conductivity, the exponent is very close to one; (3) the BNN relation [Eq. (2)] is satisfied with $p=0.59$ in the EMA and $p=0.42$ in the PPA; (4) the time-temperature superposition principle is satisfied; (5) the ac conductivity is less temperature dependent than the dc conductivity and for $n^{\prime}$ very close to one the ac conductivity becomes almost temperature independent, in particular, this always happens as $T \rightarrow 0$; (6) while $\sigma(0)$ may vary several orders of magnitude for different solids at different temperatures, the ac conductivity varies only relatively little [points (5) and (6) follow from the fact that $\Delta \epsilon$ is usually of order $\epsilon_{0}$ ]. While the universal conductivity of Eqs. (40) or (47) reproduce many observations surprisingly well, the use of log-log plot comparisons alone has rightly been warned against by Macdonald. ${ }^{106}$

Whenever the universal conductivity gives a good fit to experiments it seems that little can be learned from ac measurements. For instance, observing a power-law dependence for the real part of the conductivity with an exponent of 0.8 a few decades above the transition frequency provides no useful information about the solid un- 
der study. This is contrary to ones intuition. In 1972, Pollak and Pike ${ }^{107}$ suggested that the microscopic details of a solid are reflected in deviations from $n^{\prime}=1$. But it now appears that the situation is more complex: Details about the microscopic conduction mechanism are to be deduced from deviations from the universal conductivity curve, that itself has a nontrivial structure.

The universality seen in the macroscopic model at low temperatures is also present at low temperatures in hopping models, with an identical predicted universal frequency dependence. ${ }^{108}$ At first sight this is surprising, since hopping models are in many respects complementary to the macroscopic model: (1) hopping models are microscopic, not macroscopic; (2) they usually involve noninteracting charge carriers and ignore Coulomb interactions; (3) in hopping models one controls the local electric field while in the macroscopic model the overall potential difference is controlled; and finally (4) hopping models are stochastic while the macroscopic model is deterministic. Still, both types of models lead to large systems of sparse linear equations with coefficients that at low temperatures vary several orders of magnitude. Apparently this is enough to produce the same universality for the conductivity. Several mathematical connections exist between hopping models and resistor networks, ${ }^{10,109}$ but it is not possible to transform a hopping model into the network of Fig. 1. The Miller-Abrahams equivalent circuit for a hopping model has capacitors from each node to a voltage generator that is connected to the ground. ${ }^{47,72}$ In conclusion, ac measurements alone cannot determine what the relevant model is for conduction in a given disordered solid. To this end other measurements have to be performed ${ }^{30}$ like, e.g., transient current experiments monitoring the current after a brief laser pulse excitation, ${ }^{30,110,111}$ nonlinear conductivity measurements (the macroscopic model becomes nonlinear much earlier than hopping models), $1 / f$ noise measurements, ${ }^{112}$ or possibly Hall effect measurements. ${ }^{113}$

Some open questions remain. Is the universality seen in computer simulations as $T \rightarrow 0$ a mathematically exact fact (as believed by the author) or is there just "quasiuniversality"? It is clear that the universality is closely linked to the percolation phenomenon, but around the percolation threshold it has recently been shown ${ }^{114,115}$ (albeit in a different context than the present) that there are nonuniversal critical exponents when a broad distribution of admittances is involved. If the universality is confirmed, what is the exact form of the universal frequency dependence? Is it truly independent of the dimension? From the existence of long-time tails one expects the universal conductivity curve to be nonanalytic at $\widetilde{s}=0$. However, since the universal conductivity exists as a limit only, it is actually possible that the function is analytic and that one of Eqs. (40) or (47) is exact.

\section{ACKNOWLEDGMENTS}

The author wishes to thank P. V. Christiansen, H. Larsen, J. R. Macdonald, N. B. Olsen, I. H. Pedersen, T. Riedel, and P. Viscor for helpful comments and technical assistance. This work was supported by the Danish Natural Science Research Council.

\section{APPENDIX A: SOLVING THE EMA EQUATION}

Equation (32) may easily be solved at any temperature and real Laplace frequency. The solid curves of Fig. 3 were obtained by discretizing the equation into 3000 terms using regularly spaced energies; the conductivity was then determined at fixed $s$ and $\beta$ by the bisection method. ${ }^{97}$ Before this is done, it is convenient to rewrite Eq. (32) in terms of the variables $\widetilde{\sigma}$ and $\widetilde{s}$ defined in Eq. (39).

The universal conductivity given by Eq. (40) may be determined by means of the Newton-Raphson method. Consider first the case of real dimensionless Laplace frequencies $\widetilde{s}$. Introducing $\lambda=\ln (\widetilde{\sigma})$, Eq. (40) is rewritten by taking $\ln$ on both sides:

$$
\lambda+\ln (\lambda)=\ln (\widetilde{s}) .
$$

The Newton-Raphson method ${ }^{97}$ for solving the equation $f(\lambda)=0$ consists of iterating after the recipe

$$
\lambda_{n+1}=\lambda_{n}-\frac{f\left(\lambda_{n}\right)}{f^{\prime}\left(\lambda_{\dot{n}}\right)}
$$

In the case $f(\lambda)=\lambda+\ln (\lambda)-\ln (\widetilde{s})$, Eq. (A2) becomes

$$
\lambda_{n+1}=\lambda_{n} \frac{1-\ln \left(\lambda_{n}\right)+\ln (\widetilde{s})}{1+\lambda_{n}} .
$$

Equation (A3) is iterated until convergence by starting with, e.g., $\lambda_{1}=\widetilde{s}$ if $0<\widetilde{s}<1$ [utilizing the fact that $\lambda=\ln (\widetilde{\sigma}) \cong \widetilde{s}$ for small frequencies] and starting with, e.g., $\lambda_{1}=1$ if $\widetilde{s}>1$.

An analytic fit to $\widetilde{\sigma}(\widetilde{S})$ is the following expression:

$$
\widetilde{\sigma}(\widetilde{s})=\frac{\widetilde{s}}{\mathcal{L}_{1}(\widetilde{s})+b \mathcal{L}_{2}(\widetilde{s})+c \mathcal{L}_{3}(\widetilde{s})-(b+c) \mathcal{L}_{4}(\widetilde{s})},
$$

where $\mathcal{L}_{1}(\widetilde{s})=\ln (1+\widetilde{s})$ and one recursively defines $\mathcal{L}_{i+1}(\widetilde{s})=\ln \left[1+\mathcal{L}_{i}(\widetilde{s})\right]$. The case $b=c=0$ corresponds to the PPA solution Eq. (47). For $b=-2.2$ and $c=3.5$, Eq. (A4) provides a fit whose logarithm (base 10) for all $\widetilde{s}$ is within 0.01 of the logarithm of the true solution.

At real frequencies $\widetilde{\omega}=\widetilde{s} / i$, the EMA equation becomes complex, but it can still be solved by the NewtonRaphson method. Writing $\lambda=x+i y$, Eq. (A2) becomes

$$
x_{n+1}=x_{n}-\frac{A_{1}(n) B_{1}(n)-A_{2}(n) B_{2}(n)}{D(n)},
$$

$$
y_{n+1}=y_{n}-\frac{A_{1}(n) B_{2}(n)+A_{2}(n) B_{1}(n)}{\mathcal{D}(n)},
$$

where, if $L_{1}(n)=\ln \left(x_{n}^{2}+y_{n}^{2}\right) / 2$ and $L_{2}(n)$ $=\arctan \left(y_{n} / x_{n}\right)$, the following abbreviations have been introduced: 


$$
\begin{aligned}
& A_{1}(n)=x_{n}^{2}-y_{n}^{2}+x_{n}\left[L_{1}(n)-\ln (\widetilde{\omega})\right]+y_{n}\left(\frac{\pi}{2}-L_{2}(n)\right], \\
& A_{2}(n)=2 x_{n} y_{n}+x_{n}\left[L_{2}(n)-\frac{\pi}{2}\right]+y_{n}\left[L_{1}(n)-\ln (\widetilde{\omega})\right],
\end{aligned}
$$

$B_{1}(n)=1+x_{n}$,

$B_{2}(n)=y_{n}$,

$\mathcal{D}(n)=B_{1}{ }^{2}(n)+B_{2}{ }^{2}(n)$.

Equation (A5) is iterated until convergence starting with, e.g., $x_{1}=0.01$ and $y_{1}=\widetilde{\omega}$ if $0<\widetilde{\omega}<1 \quad$ [reflecting $\lambda=\ln (\widetilde{\sigma}) \cong i \widetilde{\omega}$ at low frequencies] and with, e.g., $x_{1}=1$ and $y_{1}=0$ if $\widetilde{\omega}>1$.

Substituting $\widetilde{s}=i \widetilde{\omega}$ into Eq. (A4) provides an analytic approximation of both $\widetilde{\sigma}^{\prime}(\widetilde{\omega})$ and $\widetilde{\sigma}^{\prime \prime}(\widetilde{\omega})$. For the real part the fit (again with $b=-2.2$ and $c=3.5$ ) has a logarithm (base 10) within 0.03 of the exact solution; for the imaginary part the logarithm of the fit is within 0.05 of the exact value for $\widetilde{\omega}>10^{-2}$ (for smaller frequencies the fit becomes poor).

\section{APPENDIX B: ACTIVATION ENERGY PROBABILITY DISTRIBUTIONS}

In the computer simulations of Ref. 14 and the present paper dimensionless activation energies were used. With the exceptions of the two Gaussian distributions the activation energies for a given probability distribution $p(E)$ were generated by utilizing the following well-known fact. If $x$ is a uniformly distributed random number between 0 and 1 and $E(x)$ is some function of $x, E$ is distributed according to the $p(E)$ given by $d p=p(E)|d E|=p(x)|d x|$. Since $p(x)=1$ one thus finds

$$
p(E)=\frac{1}{|d E / d x|} .
$$

To avoid spurious effects due to subtle correlations in system-supplied random numbers, the random numbers were generated using the function RAN0. ${ }^{97}$ This function starts by setting up an array $\operatorname{RAN}[i]$ of 97 systemsupplied random numbers. Reliable random numbers $x$ are supplied by RANO by using the given random pointer to an index $i_{y}$ between 1 and 97: RAN0 then returns $x=\operatorname{RAN}\left[i_{y}\right]$ and a new system-supplied random number is filled into the RAN array to replace $x$. Finally, $x$ is used to generate the new pointer index $i_{y}$ which is used to point out the next random number, etc.

The percolation threshold $p_{c}$ is given by $p_{c}=0.5000$ (exactly) in $2 \mathrm{D}$ and $p_{c}=0.2488$ in $3 \mathrm{D} .{ }^{81,82}$ The percolation energy $E_{c}$ that determines the dc conductivity activation energy [Eq. (19)] is found from Eq. (20):

$$
\int_{-\infty}^{E_{c}} p(E) d E=p_{c} \text {. }
$$

A knowledge of $E_{c}$ helps one to locate the frequency range of interest in the simulations; in 2D one also has $E_{g}(0)=E_{c}$ [Eq. (34)]. If $E(x)$ is an increasing function of $x$ one finds from Eqs. (B1) and (B2)

$$
E_{c}=E\left(x=p_{c}\right) \text {. }
$$

The following activation energy distributions were used in the simulations of Ref. 14 and this paper.

(1) Gaussian. From 12 random numbers $x_{i}$ (between 0 and 1), $E=\sum_{i=1}^{12} x_{i}-6$ gives a nice Gaussian distribution of $E$ with variance $1:{ }^{97}$

$$
p(E)=\frac{1}{\sqrt{2 \pi}} \exp \left[-\frac{E^{2}}{2}\right](-\infty<E<\infty) .
$$

The percolation energies are determined from Eq. (B2) that implies $\operatorname{erf}\left(-E_{c} / \sqrt{2}\right)=1-2 p_{c}$. Denoting the percolation energy in 2D by $E_{c}^{(2)}$ and in 3D by $E_{c}^{(3)}$ one finds

$$
E_{c}^{(2)}=0, \quad E_{c}^{(3)}=-0.678 .
$$

(2) Asymmetric Gaussian. For 12 random numbers one calculates $E=\left|\sum_{i=1}^{12} x_{i}-6\right|$; this generates the distribution

$$
p(E)=\sqrt{2 / \pi} \exp \left[-\frac{E^{2}}{2}\right] \quad(0<E<\infty) .
$$

Equation (B2) implies $\operatorname{erf}\left(E_{c} / \sqrt{2}\right)=p_{c} ;$ thus,

$$
E_{c}^{(2)}=0.674, \quad E_{c}^{(3)}=0.317 .
$$

(3) Cauchy. Writing $E=\tan [(\pi / 2) x]$, where $x$ is random, produces, according to Eq. (B1),

$$
p(E)=\frac{2}{\pi} \frac{1}{1+E^{2}} \quad(0<E<\infty) .
$$

Equation (B3) implies $E_{c}=\tan \left[(\pi / 2) p_{c}\right]$; thus,

$$
E_{c}^{(2)}=1, \quad E_{c}^{(3)}=0.412 .
$$

(4) Exponential. If $E=-\ln (1-x)$ one finds

$$
p(E)=e^{-E} \quad(0<E<\infty) .
$$

Equation (B3) implies $E_{c}=-\ln \left(1-p_{c}\right)$; thus,

$$
E_{c}^{(2)}=0.693, \quad E_{c}^{(3)}=0.286 .
$$

(5) Symmetric exponential. If $E= \pm \ln (1-x)$ with a random sign one finds

$$
p(E)=\frac{1}{2} e^{-|E|}(-\infty<E<\infty) .
$$

Equation (B2) implies $E_{c}=\ln \left(2 p_{c}\right)$; thus,

$$
E_{c}^{(2)}=0, \quad E_{c}^{(3)}=-0.698 .
$$

(6) Power law with exponent -4 . If $E=x^{-1 / 3}-1$ one finds 


$$
p(E)=3(1+E)^{-4} \quad(0<E<\infty) .
$$

Equation (B2) implies $E_{c}=\left(1-p_{c}\right)^{-1 / 3}-1$; thus,

$$
E_{c}^{(2)}=0.260, \quad E_{c}^{(3)}=0.100 .
$$

(7) Box. If $E=x$ one finds

$$
p(E)=1 \quad(0<E<1)
$$

and

$$
E_{c}^{(2)}=0.500, \quad E_{c}^{(3)}=0.249 .
$$

(8) Triangle. If $E=x^{1 / 2}$ one finds

$$
p(E)=2 E \quad(0<E<1) .
$$

Equation (B3) implies $E_{c}=p_{c}^{1 / 2}$; thus,

$$
E_{c}^{(2)}=0.707, \quad E_{c}^{(3)}=0.499
$$

${ }^{1}$ A. K. Jonscher, Nature 267, 673 (1977); Dielectric Relaxation in Solids (Chelsea Dielectric, London, 1983).

${ }^{2}$ A. E. Owen, J. Non-Cryst. Solids 25, 372 (1977).

${ }^{3}$ A. Mansingh, Bull. Mater. Sci. (India) 2, 325 (1980).

${ }^{4}$ A. R. Long, Adv. Phys. 31, 553 (1982).

${ }^{5}$ M. D. Ingram, Phys. Chem. Glasses 28, 215 (1987).

${ }^{6}$ S. R. Elliott, Physics of Amorphous Materials, 2nd ed. (Longman Scientific, London, 1990).

${ }^{7}$ C. A. Angell, Chem. Rev. 90, 523 (1990).

${ }^{8} \mathrm{H}$. Böttger and V. V. Bryksin, Hopping Conduction in Solids (Akademie-Verlag, Berlin, 1985).

${ }^{9}$ J. W. Haus and K. W. Kehr, Phys. Rep. 150, 263 (1987).

${ }^{10}$ J.-P. Bouchaud and A. Georges, Phys. Rep. 195, 127 (1990).

${ }^{11}$ J. O. Isard, Philos. Mag. B 62, 139 (1990); Philos. Mag. A 66, 213 (1992).

${ }^{12}$ P. Maass, J. Petersen, A. Bunde, W. Dieterich, and H. E. Roman, Phys. Rev. Lett. 66, 52 (1991).

${ }^{13}$ J. O. Isard, J. Non-Cryst. Solids 4, 357 (1970).

14J. C. Dyre, Phys. Rev. B 47, 9128 (1993).

${ }^{15}$ J. M. Stevels, in Handbuch der Physik, edited by S. Flügge (Springer-Verlag, Berlin, 1957), Vol. 20, p. 350.

${ }^{16}$ H. E. Taylor, J. Soc. Glass Tech. 41, $350 T$ (1957); 43, $124 T$ (1959).

${ }^{17}$ J. O. Isard, Proc. Inst. Elec. Eng. 109B, 440 (1962).

${ }^{18}$ M. Pollak and T. H. Geballe, Phys. Rev. 122, 1742 (1961).

${ }^{19}$ S. R. Elliott, Adv. Phys. 36, 135 (1987).

${ }^{20}$ M. Suzuki, J. Phys. Chem. Solids 41, 1253 (1980).

${ }^{21}$ A. Ya. Vinnikov and A. M. Meshkov, Fiz. Tverd Tela (Leningrad) 27, 1929 (1985) [Sov. Phys. Solid State 27, 1159 (1985)].

${ }^{22}$ A. R. Blythe, Electrical Properties of Polymers (Cambridge University Press, London, 1979).

${ }^{23}$ F. Kremer, J. Ruhe, and W. H. Meyer, Makromol. Chem., Macromol. Symp. 37, 115 (1990).

${ }^{24}$ M. P. J. van Staveren, H. B. Brom, and L. J. de Jongh, Phys. Rep. 208, 1 (1991).

${ }^{25}$ M. Sayer, A. Mansingh, J. M. Reyes, and G. Rosenblatt, J. Appl. Phys. 42, 2857 (1971).

${ }^{26}$ M. A. Careem and A. K. Jonscher, Philos. Mag. 35, 1489 (1977).

${ }^{27}$ S. Mollah, K. K. Som, K. Bose, and B. K. Chaudhuri, J. Appl. Phys. 74, 931 (1993).

${ }^{28}$ P. B. Macedo, C. T. Moynihan, and R. Bose, Phys. Chem. Glasses 13, 171 (1972).

${ }^{29}$ D. P. Almond and A. R. West, Solid State Ion. 11, 57 (1983).

30J. C. Dyre, J. Non-Cryst. Solids 135, 219 (1991).

${ }^{31}$ Impedance Spectroscopy, edited by J. R. Macdonald (Wiley, New York, 1987).

${ }^{32}$ C. A. Angell, Solid State Ion. 18-19, 72 (1986).

${ }^{33}$ K. L. Ngai, in Non-Debye Relaxation in Condensed Matter, edited by T. V. Ramakrishnan and M. R. Lakshmi (World
Scientific, Singapore, 1987), p. 23.

${ }^{34}$ L. A. Dissado and R. M. Hill, Phys. Rev. B 37, 3434 (1988).

${ }^{35}$ G. A. Niklasson, J. Appl. Phys. 62, R1 (1987).

${ }^{36}$ S. Yoshikado, T. Ohachi, I. Taniguchi, Y. Onodo, M. Watanabe, and Y. Fujiki, Solid State Ion. 7, 335 (1982).

${ }^{37}$ A. J. Epstein, in Handbook of Conjugated Electrically Conducting Polymers, edited by T. A. Skotheim (Marcel Dekker, New York, 1986), Vol. 2, p. 1041.

${ }^{38}$ J. L. Barton, Verres Réfr. 20, 328 (1966).

${ }^{39}$ T. Nakajima, 1971 Annual Report, Conference on Electric Insulation and Dielectric Phenomena (National Academy of Sciences, Washington, D.C., 1972), p. 168.

${ }^{40}$ H. Namikawa, J. Non-Cryst. Solids 18, 173 (1975).

${ }^{41} \mathbf{M}$. Tomozawa, in Treatise on Materials Science, edited by $\mathbf{M}$. Tomozawa (Academic, New York, 1977), Vol. 12, p. 283.

42J. C. Dyre, J. Non-Cryst. Solids 88, 271 (1986).

${ }^{43}$ H. Scher and M. Lax, Phys. Rev. B 7, 4491 (1973); 7, 4502 (1973).

${ }^{44}$ S. Summerfield, Philos. Mag. B 52, 9 (1985).

45J. C. Dyre, J. Appl. Phys. 64, 2456 (1988).

${ }^{46}$ C. J. F. Bottcher, O. C. Van Belle, P. Bordewijk, and A. Rip, Theory of Electric Polarisation (Elsevier, Amsterdam, 1990), Vol. 2.

${ }^{47}$ A. Miller and E. Abrahams, Phys. Rev. 120, 745 (1960).

${ }^{48}$ I. G. Austin and N. F. Mott, Adv. Phys. 18, 41 (1969).

${ }^{49}$ S. R. Elliott, Philos. Mag. 36, 1291 (1977).

${ }^{50}$ I. M. Hodge, M. D. Ingram, and A. R. West, J. Electroanal. Chem. 74, 125 (1976).

51 J. R. Macdonald, J. Appl. Phys. 62, R51 (1987).

${ }^{52}$ R. Stumpe, Phys. Status Solidi A 88, 315 (1985).

${ }^{53}$ R. Blender and W. Dieterich, J. Phys. C 20, 6113 (1987).

${ }^{54}$ A. Coniglio, M. Daoud, and H. J. Herrmann, J. Phys. A 22, 4189 (1989).

${ }^{55}$ P. Smilauer, Thin Solid Films 203, 1 (1991).

${ }^{56}$ B. E. Springett, Phys. Rev. Lett. 31, 1463 (1973).

${ }^{57}$ I. Webman, J. Jortner, and M. H. Cohen, Phys. Rev. B 15, 5712 (1977).

58J. Sinkkonen, Phys. Status Solidi B 103, 231 (1981).

${ }^{59}$ I. I. Fishchuk, Phys. Status Solidi A 93, 675 (1986).

${ }^{60}$ L. A. Dissado and R. M. Hill, Proc. R. Soc. London, Ser. A 390, 131 (1983).

${ }^{61}$ K. L. Ngai and F.-S. Liu, Phys. Rev. B 24, 1049 (1981).

${ }^{62}$ K. Weron, J. Phys.: Condens. Matter. 3, 9151 (1991).

${ }^{63}$ S. Havlin and D. Ben-Avraham, Adv. Phys. 36, 695 (1987).

${ }^{64}$ A. K. Jonscher, Appl. Phys. A 55, 135 (1992).

${ }^{65}$ R. Kubo, J. Phys. Soc. Jpn. 12, 570 (1957).

66J. C. Kimball and L. W. Adams, Phys. Rev. B 18, 5851 (1978).

${ }^{67}$ T. Odagaki and M. Lax, Phys. Rev. B 24, 5284 (1981).

${ }^{68}$ J. K. E. Tunaley, Phys. Rev. Lett. 33, 1037 (1974).

${ }^{69}$ V. V. Bryksin, Fiz. Tverd. Tela (Leningrad) 22, 2441 (1980) [Sov. Phys. Solid State 22, 1421 (1980)]. 
${ }^{70}$ S. Summerfield, Solid State Commun. 39, 401 (1981).

${ }^{71}$ B. Movaghar and W. Schirmacher, J. Phys. C 14, 859 (1981).

${ }^{72}$ S. Summerfield and P. N. Butcher, J. Phys. C 15, 7003 (1982).

${ }^{73}$ B. I. Shklovskii and A. L. Efros, Electronic Properties of Doped Semiconductors (Springer-Verlag, Berlin, 1984).

${ }^{74}$ N. W. Ashcroft and N. D. Mermin, Solid State Physics (Holt, Rinehart, and Winston, New York, 1976), Appendix K.

${ }^{75}$ B. Pistoulet, F. M. Roche, and S. Abdalla, Phys. Rev. B 30, 5987 (1984).

${ }^{76}$ C. J. Adkins, J. Phys. C 20, 235 (1987).

${ }^{77}$ S. Kirkpatrick, Rev. Mod. Phys. 45, 574 (1973).

${ }^{78}$ V. Ambegaokar, B. I. Halperin, and J. S. Langer, Phys. Rev. B 4, 2612 (1971).

${ }^{79}$ B. I. Shklovskii and A. L. Efros, Zh. Eksp. Teor. Fiz. 60, 867 (1971) [Sov. Phys. JETP 33, 468 (1971)].

${ }^{80}$ D. Berman, B. G. Orr, H. M. Jaeger, and A. M. Goldman, Phys. Rev. B 33, 4301 (1986).

${ }^{81}$ H. Kesten, Percolation Theory for Mathematicians (Birkhauser, Boston, 1982).

${ }^{82}$ D. B. Gingold and C. J. Lobb, Phys. Rev. B 42, 8220 (1990).

${ }^{83}$ S. Tyc and B. I. Halperin, Phys. Rev. B 39, 877 (1989).

${ }^{84}$ R. Landauer, in Electrical Transport and Optical Properties of Inhomogenous Media (Ohio State University, 1977), Proceedings of the First Conference on the Electrical Transport and Optical Properties of Inhomogeneous Materials, AIP Conf. Proc. No. 40, edited by J. C. Garland and D. B. Tanner (AIP, New York, 1978), p. 2.

${ }^{85}$ J. A. Krumhansl, in Amorphous Magnetism, edited by H. O. Hooper and A. M. de Graaf (Plenum, New York, 1973), p. 15.

${ }^{86} \mathrm{~F}$. Yonezawa, in The Structure and Properties of Matter, edited by T. Matsubara (Springer-Verlag, Berlin, 1982), p. 383.

${ }^{87}$ B. D. Hughes, M. Sahimi, L. E. Scriven, and H. T. Davis, Int. J. Eng. Sci. 22, 1083 (1984).

${ }^{88}$ J. Koplik, J. Phys. C 14, 4821 (1981).

${ }^{89}$ M. Hori and F. Yonezawa, J. Phys. C 10, 229 (1977).

${ }^{90}$ J. M. Luck, Phys. Rev. B 43, 3933 (1991).

91 J. Bernasconi, W. R. Schneider, and H. J. Wiesmann, Phys. Rev. B 16, 5250 (1977).
${ }^{92}$ M. Sahimi, Chem. Eng. Commun. 64, 177 (1988).

${ }^{93} \mathrm{D}$. Stauffer and A. Aharoni, Introduction to Percolation Theory, 2nd ed. (Taylor and Francis, London, 1992); M. B. Isichenko, Rev. Mod. Phys. 64, 961 (1992).

${ }^{94}$ S. Tyc and B. I. Halperin, Physica A 157, 210 (1989).

${ }^{95}$ R. Fogelholm, J. Phys. C 13, L571 (1980).

${ }^{96}$ D. J. Frank and C. J. Lobb, Phys. Rev. B 37, 302 (1988).

${ }^{97}$ W. H. Press, B. P. Flannery, S. A. Teukolsky, and W. T. Vetterling, Numerical Recipes (Cambridge University Press, Cambridge, 1986).

${ }^{98}$ K. Stuben, Appl. Math. Computation 13, 419 (1983).

${ }^{99}$ A. Brandt, Appl. Math. Computation 19, 23 (1986).

${ }^{100}$ J. Goodman and A. D. Sokal, Phys. Rev. D 40, 2035 (1989).

${ }^{101}$ B. Derrida and J. Vannimenus, J. Phys. A 15, L557 (1982).

${ }^{102}$ G. G. Batrouni, A. Hansen, and M. Nelkin, Phys. Rev. Lett. 57, 1336 (1986).

${ }^{103}$ The AMG1R5 FORTRAN subroutine is available from "casper.na.cs.yale.edu" (128.36.12.1); $\log$ in as anonymous ftp, then look for the file "amg.tar.Z" in the "gmd" directory under the "mgnet" directory.

${ }^{104}$ R. G. Edwards, J. Goodman, and A. D. Sokal, Phys. Rev. Lett. 61, 1333 (1988).

${ }^{105}$ Electrical Transport and Optical Properties of Inhomogeneous Media (Ref. 84); Physica A 157, 1 (1989).

106J. R. Macdonald, J. Appl. Phys. 65, 4845 (1989).

${ }^{107}$ M. Pollak and G. E. Pike, Phys. Rev. Lett. 28, 1449 (1972).

108J. C. Dyre (unpublished).

${ }^{109}$ P. G. Doyle and J. L. Snell, Random Walks and Electric Networks (The Mathematical Association of America, Washington, D.C., 1984).

${ }^{110}$ P. N. Butcher, Philos. Mag. B 37, 653 (1978).

${ }^{111}$ V. V. Bryksin, Fiz. Tverd. Tela (Leningrad) 25, 2431 (1983) [Sov. Phys. Solid State 25, 1395 (1983)].

${ }^{112}$ R. Rammal, C. Tannous, and A.-M. S. Tremblay, Phys. Rev. A 31, 2662 (1985).

${ }^{113}$ I. I. Fishchuk, J. Phys.: Condens. Matter 4, 8045 (1992).

${ }^{114}$ W. Dieterich, Philos. Mag. B 59, 97 (1989).

${ }^{115}$ M. Octavio and C. J. Lobb, Phys. Rev. B 43, 8233 (1991). 